\title{
The role of vertically varying cloud fraction in the parametrization of microphysical processes in the ECMWF model
}

\author{
By Christian Jakob ${ }^{1 *}$ and Stephen A. Klein ${ }^{2}$ \\ ${ }^{1}$ European Centre for Medium-Range Weather Forecasts, UK \\ ${ }^{2}$ Geophysical Fluid Dynamics Laboratory/NOAA, USA
}

Received 17 March 1998; revised 20 July 1998

SUMMARY

Global Circulation Models (GCMs) have generally treated only the radiative impacts of vertically varying cloud fraction by use of a cloud overlap assumption. In this study, the microphysical impacts of vertically varying cloud fraction are addressed by developing a sub-grid scale precipitation model which resolves the vertical variation of cloud fraction. This sub-grid model subdivides the grid boxes into homogeneous columns which are either completely clear or cloudy. By comparing the columnaveraged microphysical quantities from the sub-grid scale precipitation model to the parametrization in the ECMWF model, the ability of the ECMWF model to account for the sub-grid nature of cloud and precipitation microphysics is assessed. It is found that the ECMWF model overestimates precipitation evaporation in the tropical mid-troposphere. This results from (a) an incorrect parametrization of the area of the grid box covered by precipitation, and (b) the inadequacy of assuming a single value for the precipitation rate in the grid box.

In addition to assessing the ability of the ECMWF model to parametrize the sub-grid nature of cloud microphysics, the subgrid precipitation model is used to show that the cloud overlap assumption has a large impact on the evaporation of precipitation. In light of the current trend towards more sophisticated cloud and precipitation parametrization in GCMs, more attention should be paid to the impact of vertical cloud fraction variations on the parametrized microphysics.

\section{INTRODUCTION}

The representation of clouds in atmospheric general circulation models has evolved considerably over the last ten years. Almost all global models now include parametrizations that describe the budget of the cloud condensate for each of the model's vertical levels with a prognostic equation (e.g., Sundqvist 1978, Smith 1990, Tiedtke 1993, Fowler et al. 1996, Del Genio et al. 1996). The predicted cloud condensate is then used for radiation calculations, thereby coupling the cloud radiative forcing and latent heat release associated with the condensation and evaporation processes.

Besides parametrizing the density of cloud condensate many parametrizations also describe in some form the fraction of a GCM grid-box covered by clouds (Sundqvist 1978, Smith 1990, Tiedtke 1993). Cloud fraction parametrizations of this kind represent in a simple way the complex structure of cloud fields both in the horizontal and in the vertical. Figure 1 is a schematic of a distribution of clouds frequently encountered in tropical convective situations (e.g., Houze and Betts 1981) where penetrating convective towers with their associated anvils and outflow cirrus coexist with shallow or medium convective clouds. One may ask if a cloud parametrization scheme should be able to resolve some of the variability shown in Figure 1 by producing vertically varying cloud fraction and condensate.

Vertical variations in cloud fraction and optical thickness influence the distribution of the radiative fluxes in the atmosphere. Radiation parametrization schemes account for this variability by introducing overlap assumptions (e.g., Geleyn and Hollingsworth 1979, Morcrette and Fouquart 1986), which determine the horizontal position of a 'cloud' at each model level relative to the clouds at other model levels. Yu et al. (1996) have used these overlap assumptions to divide model grid boxes into sub-columns when comparing model clouds to satellite observations. Stubenrauch et al. (1997) recently proposed an overlap scheme which creates blocks of cloud spanning several model levels in the vertical and then distributes the blocks in the horizontal following some overlap rules.

The effects of vertical variations of cloud fraction on the parametrization of microphysical processes have received less attention than their radiative effects. For instance, if ice from a cirrus anvil falls into a cloud with supercooled liquid (as depicted in the leftmost low cloud in Figure 1), then the liquid may be converted to ice through the Bergeron process (Baker 1997). Whether or not this occurs depends on whether the anvil ice falls into the lower cloud. Similarly evaporation of precipitation can only occur when precipitation falls into clear air. GCMs have shown a large sensitivity to the treatment of precipitation evaporation (e.g., Gregory 1995) and it therefore appears to be desirable to treat the sub-grid scale nature of the evaporation process more carefully. Few studies have tried to account for such effects by determining a precipitation fraction (Rotstayn 1997) or by adjusting microphysical parameters such as autoconversion rates and accretion rates in case of vertically varying cloud (Bechtold et al. 1993).

* corresponding author: ECMWF, Shinfield Park, Reading, RG2 9AX, UK 


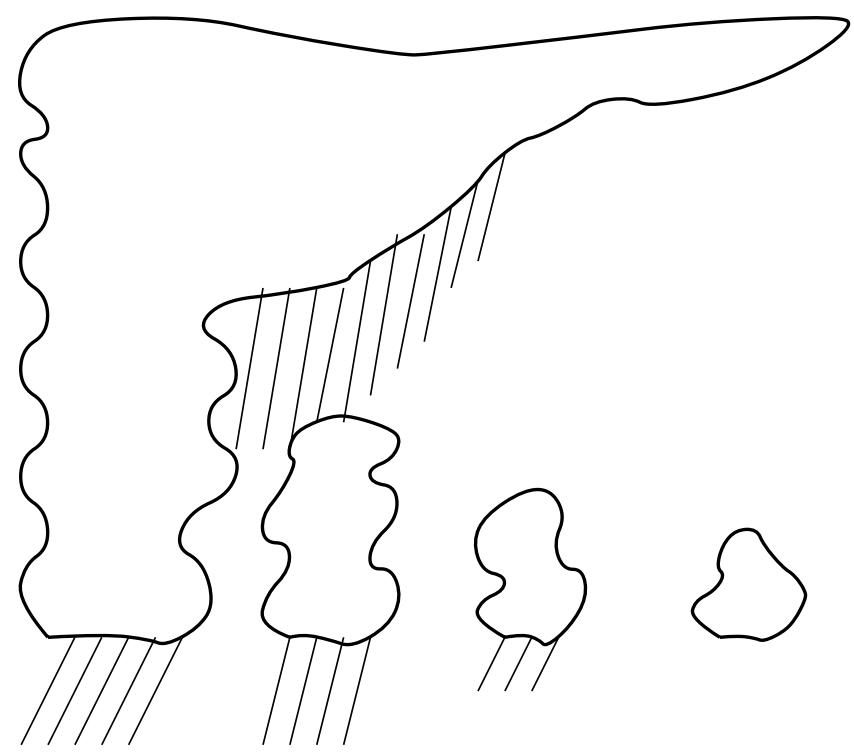

Figure 1. Schematic of a possible cloud distribution in the Tropics

This exploratory study investigates the micro- and macrophysical impact of an explicit treatment of vertical variation of cloud fraction in the European Centre for Medium-Range Weather Forecasts (ECMWF) cloud scheme (Tiedtke 1993, Jakob 1994). The technique applied is to horizontally divide each grid box at each level into a number $N$ of smaller "sub-boxes". Each of the sub-boxes is assigned to be either completely cloudy or completely clear depending on the cloud fraction predicted for the whole grid box by the cloud parametrization and on the cloud overlap assumption used by the radiation parametrization. This yields $N$ vertical columns of sub-boxes with up to $N$ different vertical distributions of cloud. The microphysics parametrization is then calculated separately for each column of sub-boxes. The relevant grid-mean quantities (e.g., precipitation fluxes, evaporation rates etc.) are computed by averaging over all sub-boxes. Comparison of the results to the original parametrization provides an indication of the sub-grid scale effects of cloud microphysics.

Section 2 describes the current parametrization; the sub-grid scale precipitation model is introduced in section 3. Section 4 compares the results of the more complex treatment to the current parametrization and discusses the main differences. A summary and discussion of the results is given in section 5 .

\section{The 1993 ECMWF ClOUd SCHEME}

The 1993 ECMWF cloud scheme is described in detail in Tiedtke (1993) (hereafter referred to as T93) with some changes listed in Jakob (1994). The scheme is based on two prognostic equations for cloud fraction $a$ and cloud condensate $l$ (the sum of water and ice), i.e.,

$$
\frac{\partial a}{\partial t}=A_{a}+S_{a}-D_{a}
$$

and

$$
\frac{\partial l}{\partial t}=A_{l}+S_{l}-D_{l}
$$

where $A_{a, l}$ represents advection and $S_{a, l}$ and $D_{a, l}$ are the source and dissipation terms for cloud fraction and condensate respectively. Sources for cloud fraction and condensate in the scheme arise from convection, boundary layer turbulence in case of stratocumulus clouds, large-scale lifting and diabatic cooling. The dissipation terms are determined by evaporation processes due to subsiding motions including both large-scale subsidence and cumulus-induced subsidence, diabatic heating, turbulent erosion processes at both cloud top and cloud sides and by precipitation processes in the case of condensate.

The parametrization of microphysical processes is as described in Tiedtke (1993) and Jakob (1994) throughout this study. The generation of precipitation is dependent on the phase of the condensate, which is distinguished between pure ice, mixed phase and pure water by a quadratic function of temperature varying from pure ice 
below $-23^{\circ} \mathrm{C}$ to pure water above $0^{\circ} \mathrm{C}$. For pure ice clouds, the mean terminal fall velocity of ice particles is prescribed as a function of ice content following Heymsfield and Donner (1990). In mixed phase and water clouds Sundqvist's (1988) conversion scheme is used. The evaporation of rain and snow follows the formulation of Kessler (1969) and the melting of snow is parametrized by allowing a layer to cool to the melting point $\left(0^{\circ} \mathrm{C}\right)$ over a timescale of 5 hours. The numerical treatment is implicit for the conversion from cloud water/ice to precipitation (Tiedtke 1993) and explicit for melting and evaporation.

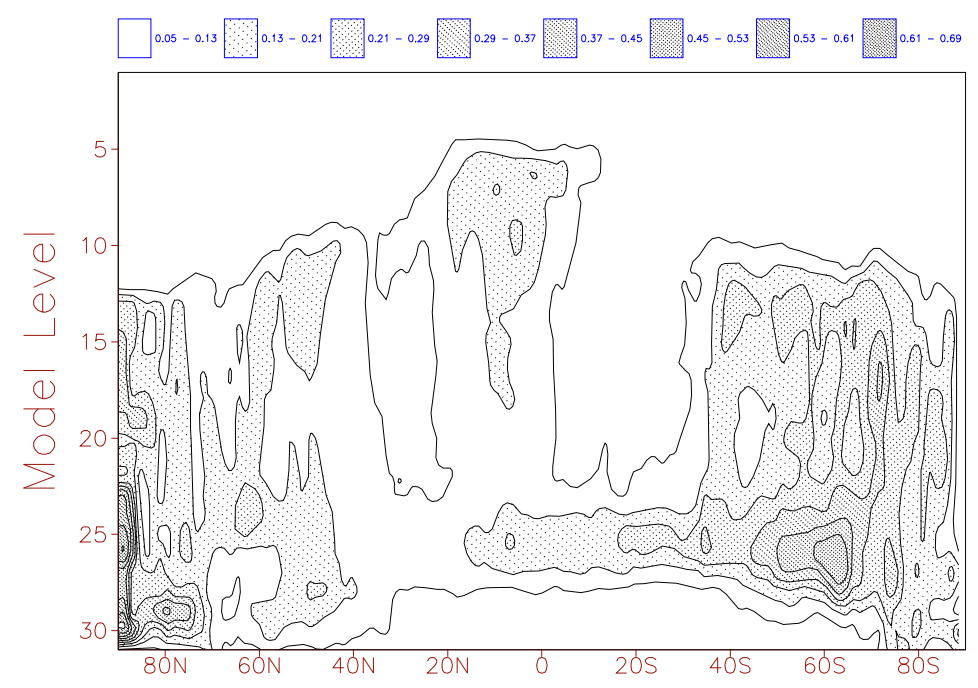

Figure 2. Zonal mean of cloud fraction in the ECMWF model for 1 July 1997, 12UTC. Altitude increases with decreasing model level number. The relation of the model levels to pressure is depicted in Figure 3.

As suggested by the schematic diagram in Figure 1 large vertical variations in cloud fraction can exist in nature over an area the size of a GCM grid box. Before investigating whether the representation of these effects in the ECMWF model is of importance it is necessary to establish if the model produces significant vertical variations in cloud fraction. Figure 2 shows the zonal mean cloud distribution as a function of model level in the operational model for 1 July 1997, 12 UTC. Zonal mean cloud fraction varies substantially in the vertical in the model. However, in the zonal mean such a variation could be caused by the occurrence of clouds at different grid points in the horizontal and does not necessarily imply strong vertical variations at any given location. Figure 3 shows a cloud fraction profile for a single point in the Tropics $\left(2^{\circ} \mathrm{N}, 160^{\circ} \mathrm{E}\right)$ that was randomly chosen. It is evident that instantaneous local cloud fields produced by the ECMWF scheme can indeed have large vertical variations of cloud fraction. The cloud fields depicted in Figures 2 and 3 will form the basis of the investigations in the following sections.

\section{The SUB-GRID SCALE PRECIPITATION MODEL}

Bulk microphysical parametrizations (e.g., Hsie et al. 1980, Lin et al. 1983, Rutledge and Hobbs 1983) describing the formation and evaporation of different precipitation species are typically formulated such that they are applicable on small scales in regions that are either totally clear or cloudy. Their application in large volumes such as a GCM grid box which can contain both cloudy and cloud free areas is therefore not straightforward. Several approaches to this problem have been proposed. The simplest approach is to ignore partial cloudiness and assume a cloud fraction of one whenever condensate occurs in a grid box (e.g., Fowler et al. 1996). Schemes that parametrize cloud fraction often use in microphysical calculations the in-cloud water content $l_{c}=l / a$ where $l$ is the grid mean water/ice content and $a$ is the cloud fraction (Tiedtke 1993). Until very recently (Bechthold et al. 1993, Tiedtke 1993, Rotstayn 1997), the rather obvious fact that partial cloud fraction yields precipitation which only covers a fraction of the grid box has been ignored. 


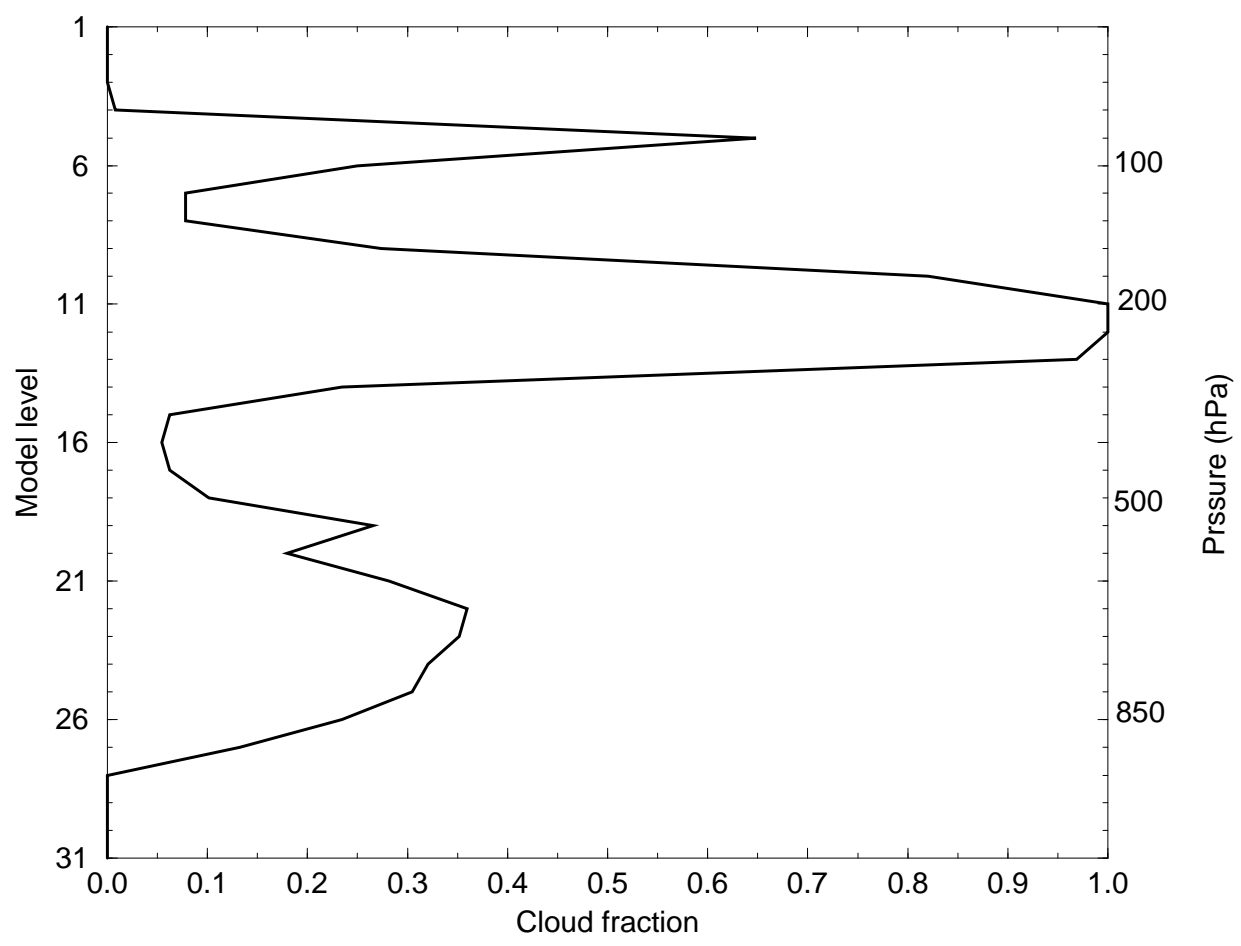

Figure 3. Vertical distribution of cloud fraction at a single point in the Tropics

In order to systematically assess the effects of partial cloudiness and partial precipitation coverage a sub-grid scale precipitation representation is developed. The basic idea is to subdivide each grid box into $N$ sub-columns ( $N=20$ is used for most of this study) in which the cloud fraction is assigned to be zero or one at every model level. The microphysical parametrization is then applied to each of the sub-columns and the relevant grid-mean quantities (e.g., precipitation flux, melting and evaporation rates) are calculated by summing up the values over all sub-columns. This is equivalent to an increased horizontal resolution for the microphysical calculations. A possible distribution of cloudy and clear sky sub-columns for the distribution of cloud fraction in Figure 3 is shown in Figure 4. To arrive at the distribution of cloudy and clear-sky sub-columns shown several assumptions were made, the details of which are explained below.

Firstly it is assumed that clouds completely fill the grid box in the vertical; i.e., the fraction of grid box volume that contains cloud is equal to the fraction of the horizontal area of a grid box that contains cloud. Although many clouds have thicknesses less than 500 meters (Wang and Rossow 1995), this may not be too bad an approximation for the ECMWF model which has 31 levels in the vertical with typical resolution of 40 $\mathrm{hPa}$ (about 400 to $700 \mathrm{~m}$ ) in the troposphere.

Secondly, at each level the specification of which sub-columns contain cloud is entirely consistent with the cloud overlap assumption used for the sub-grid scale flux calculations in the radiation scheme. The overlap assumption currently used in the radiation scheme is that of maximum-random overlap (Geleyn and Hollingsworth 1979, Morcrette and Fouquart 1986). It can be described using the following equation which specifies the total horizontal area, $C^{k}$, covered by clouds between the top of the atmosphere and a given model level $k$ as :

$$
\frac{1-C^{k}}{1-C^{k-1}}=\frac{1-\max \left(a^{k-1}, a^{k}\right)}{1-\min \left(a^{k-1}, 1-\delta\right)}
$$

where $a^{k}$ is the cloud fraction of level $k, \delta=10^{-6}$, and $k=1$ for the top model level. $C^{0}$ and $a^{0}$ are set to zero. This equation yields random overlap for clouds which do not occur in adjacent vertical levels but maximum overlap if clouds occur at adjacent levels with cloud fraction monotonically increasing or decreasing with height. This is broadly consistent with the data on cloud overlap of Tian and Curry (1989). At each level, the number 


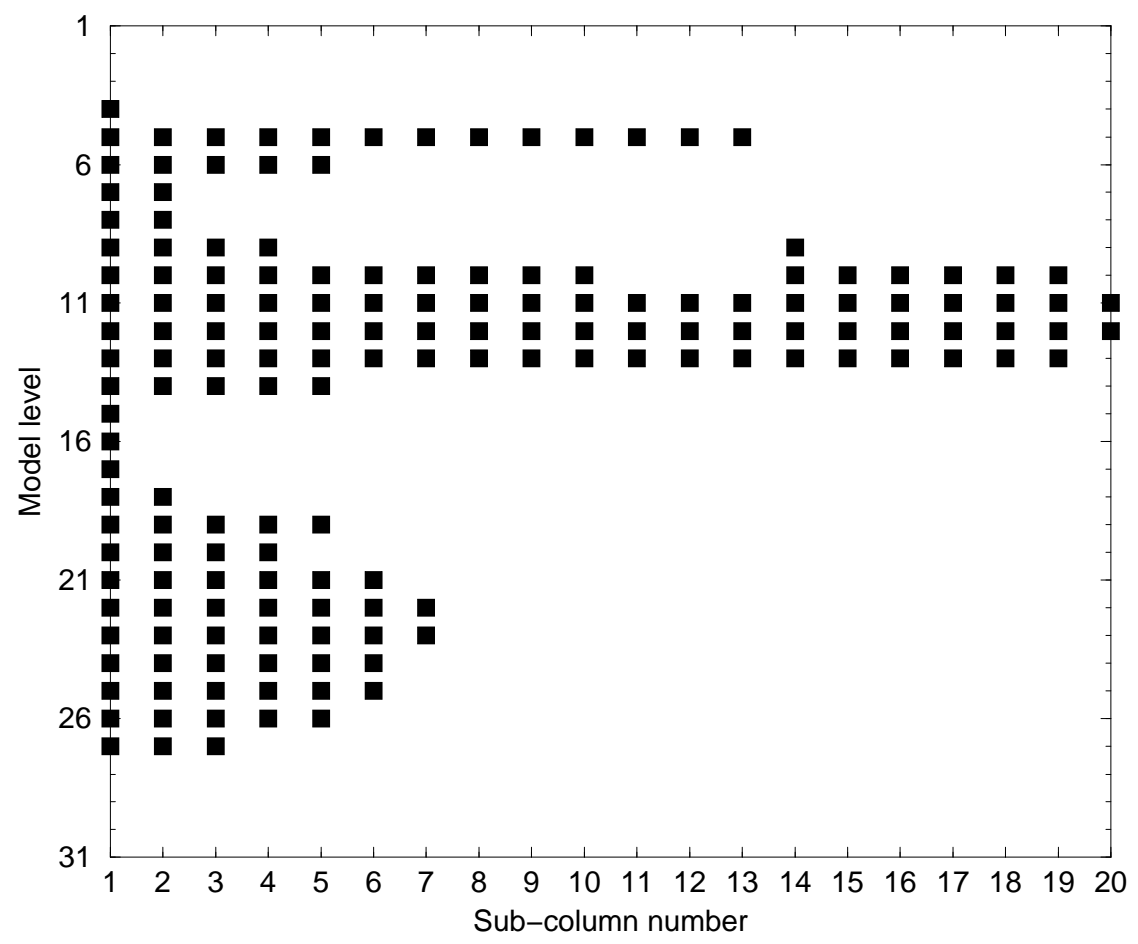

Figure 4. Arrangement of cloudy (black squares) and clear-sky columns for the cloud fraction profile in Figure 3 following the sub-grid scale algorithm

of sub-columns that contain cloud is defined to be the nearest integer value of $20 \cdot a^{k}$.

The use of equation (3) provides a total cloud cover, $C^{k}$, by applying it from the model top to level $k$ given the distribution of clouds layer by layer. However the knowledge of $C^{k}$ and $a^{k}$ alone is not sufficient to unambiguously assign the distribution of cloudy sub-columns. In Figure 4 it is for instance obvious that $C_{k}$ does not change below model level 11, where it reaches a value of 1 . Hence, the five cloudy sub-columns in level 14 , where $a^{14} \approx 0.25$, could be placed in any sub-box without violating (3). Therefore, two additional assumptions are made: i) in the spirit of maximum overlap for clouds in adjacent levels, clouds are assigned to those sub-columns which contain cloud in the layer immediately above in preference to those sub-columns which do not contain cloud in the layer immediately above but do have clouds higher in the same sub-column, and ii) the assignment of cloudy boxes begins from the sub-column furthest to the "left" that fulfills i). Since especially ii) is rather arbitrary, sensitivity tests to the placement of cloudy sub-columns will be carried out in a later section. The "left" assumption is used as the default assumption for the rest of this study because this is the cloud placement that is implicitly assumed in the T93 scheme.

The amount of liquid and ice at each level is calculated over the mean cloudy area of the grid box as described in Tiedtke (1993). It must then be divided among the cloudy sub-columns of each level. For want of a better method, each cloudy sub-column is assigned the same amount of liquid and ice assuming a constant in-cloud water/ice content defined as

$$
l_{c, i n t}^{k}=\frac{l^{k}}{a^{k} \text { int }},
$$

where $l^{k}$ is the grid mean liquid water/ice content and $a^{k}{ }_{\text {int }}$ is a rounded cloud fraction calculated as the fraction of sub-boxes at each level that contain cloud. It is necessary to use a rounded cloud fraction in the definition of $l_{c, \text { int }}^{k}$ in order to conserve water. After the allocation of the condensed water in the sub-columns, the same microphysical formulae used for the original model are applied to each sub-column separately. That is, for each sub-box the generation and evaporation of precipitation is calculated at each level. Averaging 
over sub-columns yields grid-mean quantities (precipitation and evaporation rates) that can be compared to the T93 parametrization. In all calculations a homogeneous distribution of temperature at the beginning of the microphysical calculations is assumed, i.e. each sub-column has the same temperature initially. Through melting and evaporation of precipitation the temperature will change differently in each sub-column. The new grid-mean temperature is calculated from the grid-mean melting and evaporation rates that are calculated by averaging over their values in the individual sub-columns.

In applying the microphysical formulae to the sub-columns two changes are necessary. The first change is to the melting of snow. In the current model, the amount of melting is limited such that the whole grid box would be cooled back to the freezing temperature over a time scale $\tau=5 h$, even if precipitation covers only a small fraction of the grid box. For the sub-grid scale precipitation model the amount of melting is limited such that only the sub-column in which melting occurs can be cooled to the freezing temperature. This implies that if the fraction of the grid box covered by precipitation is less than unity, the energy available for melting is smaller in the sub-grid scale precipitation parametrization than in T93. The main effect of this difference is to spread melting further in the vertical.

The second change is to the evaporation of precipitation. The formula for evaporation of precipitation depends in part on the humidity of the air into which the precipitation is evaporating. Instead of using the grid mean humidity in the formula as is done in the current parametrization, an estimate of the humidity of the clear portion of the grid box is calculated using the cloud fraction and the grid-mean humidity. Assuming that the temperature inside the cloud is the same as the grid mean temperature, the grid-mean specific humidity $\left(q_{v}\right)$ is the sum of the saturation value at the grid-mean temperature $\left(q_{s}\right)$ in the cloudy portion of the grid and a mean clear sky value humidity $\left(q_{v}{ }^{c l r}\right)$ :

$$
q_{v}=a q_{s}+(1-a) q_{v}^{c l r}
$$

To calculate the evaporation of precipitation in the sub-grid precipitation model it is assumed that each cloud-free sub-column has a value of specific humidity equal to the value of $q_{v}{ }^{c l r}$ which satisfies (5).

In implementing the sub-grid scale precipitation model, special treatment was given to those grid boxes which have a cloud fraction less than one-half of the size of a sub-column (i.e., $a^{k}<0.025$ for a model with 20 sub-columns). Normally, the rounding of cloud fraction to the nearest sub-column would assign all sub-columns as clear sky and no precipitation could be generated from clouds with these small cloud fractions. In order to avoid problems of that nature, it is required that if the cloud fraction is greater than zero then at least one sub-box in layer $k$ must be filled with cloud. Water conservation in this case is ensured by redistributing the water quantities over the whole sub-box.

It is worthwhile pointing out that the distribution of the cloudy columns in Figure 4 should not be interpreted as a spatially contiguous distribution. Shifting all columns randomly, treating each one as a whole in the vertical, will by construction not change the results of the simulation since each column is treated as an independent quantity. It is therefore better to think of the distribution of the cloudy columns as a probability state given the set of rules outlined above.

\section{COMPARISON OF THE SUB-GRID SCALE PRECIPITATION MODEL WITH THE ORIGINAL SCHEME}

\section{(a) Single grid-point simulations}

Both the sub-grid scale precipitation model and the current model are used to simulate the precipitation processes at a single grid-point. To assess the direct effects of the sub-grid model on precipitation related processes only the first timestep of the integration is considered, with all other physical processes switched off. Hence, the simulation is entirely governed by the initial profiles of cloud cover, cloud water/ice, humidity and the thermodynamic variables. The profiles used are those of the tropical case in Figure 3. The initial profile of grid-mean cloud water/ice is shown in Figure 5. The timestep used is 15 minutes which is a typical value used in ECMWF's T213L31 model.

The aim of the study is to assess the impact of variations in cloud fraction on cloud microphysics. For this purpose the sub-grid model can be regarded as a higher resolution model, which yields more accurate values of precipitation. The current parametrization should approximate the sub-grid model results if it were to properly account for the sub-grid scale variability of cloud and precipitation. Many factors, including a poor microphysical formulation itself, may cause the results of the sub-grid model to be far from reality but only the differences between the two simulations are of interest for the sensitivity study which is the purpose of this work. 


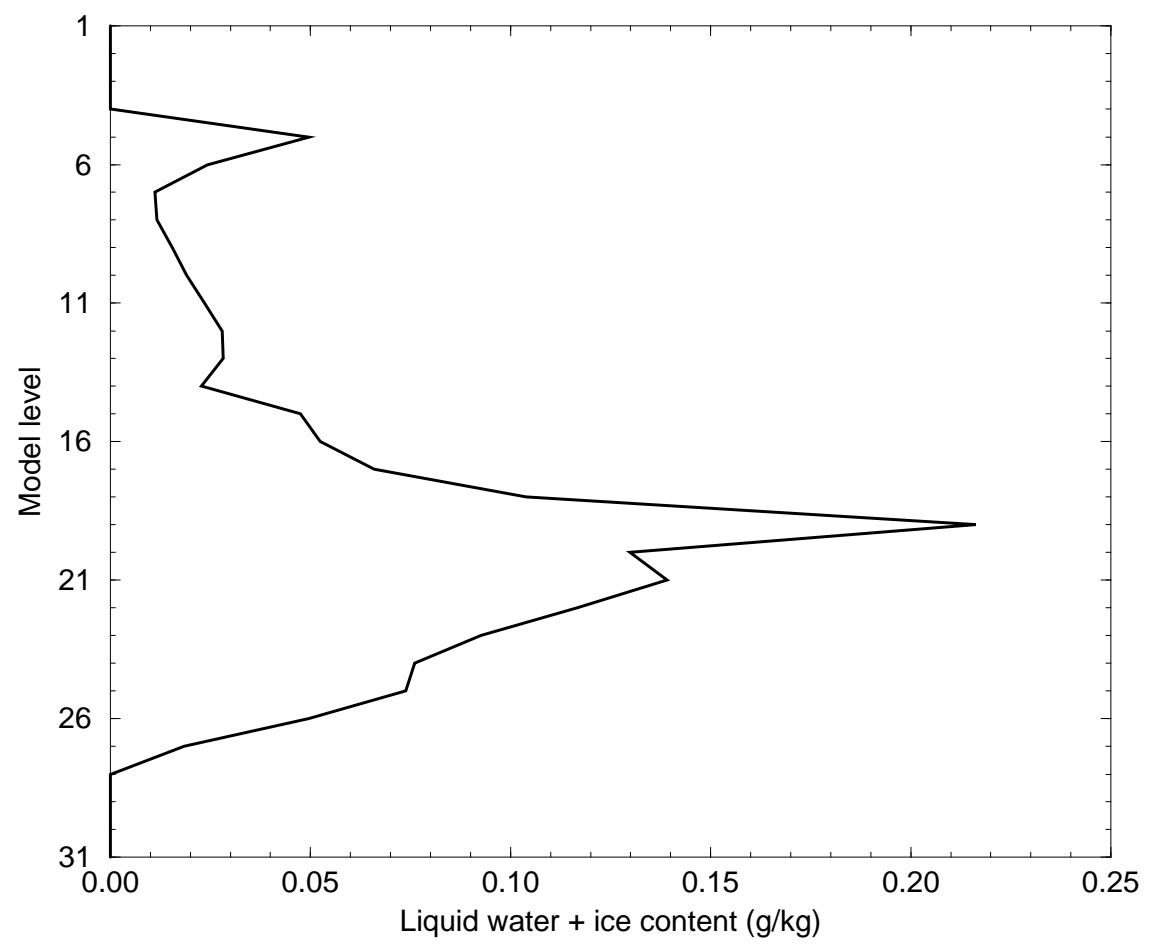

Figure 5. Vertical distribution of cloud liquid water plus ice in the single column model profile shown in Figure 3

Using the distribution of cloudy and clear-sky sub-columns shown in Figure 4 the microphysical part of the cloud scheme is integrated separately for each of the sub-columns. A large variation is found in the precipitation fluxes between the different sub-columns. For example, Figure 6 shows the vertical distribution of precipitation for sub-columns one and six. Sub-column one, by construction, contains the maximum number of cloudy levels. Hence, precipitation falls through clouds from model level 5 down to model level 27, leading to large amounts of accretion and no evaporation above cloud base. As a consequence the precipitation rate reaches a maximum of more than $120 \mathrm{~mm}$ /day just above cloud base. In contrast sub-column six contains three cloud layers, extending over one, four and five model levels respectively, separated by layers of clear sky. Precipitation forms first in model level 5. Although levels 6 through 9 contain unsaturated air, the precipitation rate remains constant until the next cloudy layer (model level 10). No sublimation occurs between levels 6 and 9 because the T93 scheme assumes that precipitation cannot evaporate when the relative humidity exceeds a critical value of 80 $\%$. After leaving the base of the second cloud layer evaporation of precipitation results in the total depletion of the precipitation at level 17 so that no precipitation reaches the lowest cloud layer with its top at level 21 . This is an example of the importance of the seeder-feeder mechanism: no precipitation reaches the low cloud from above so that only the warm-phase microphysics is important for the development of the lowest cloud layer. The precipitation reaching the surface in this sub-column (slightly more than $2 \mathrm{~mm} /$ day) is entirely due to conversion processes in the lowest cloud layer and has been reduced by evaporation in the sub-cloud layer.

By counting the sub-columns that contain precipitation at each level it is possible to derive the fraction of the grid box covered by precipitation (from now on referred to as precipitation fraction, $a_{P}$ ). This parameter is used in several ways in existing parametrization schemes (Bechthold et al. 1993, Tiedtke 1993, Rotstayn 1997). For example the evaporation of precipitation in model level $k$ in the T93 parametrization is described as

$$
E_{P}{ }^{k}=\max \left(a_{P}{ }^{k}-a^{k}, 0\right) \cdot 5.44 \cdot 10^{-4}\left(q_{s}{ }^{k}-q^{k}\right)\left[\left(\frac{p^{k}}{p_{s}}\right) \frac{1}{5.9 \cdot 10^{-3}} \frac{P^{k}}{a_{P}{ }^{k}}\right]^{0.577} .
$$

The precipitation fraction, $a_{P}{ }^{k}$, appears twice in this equation. Firstly it is used to calculate the area over 

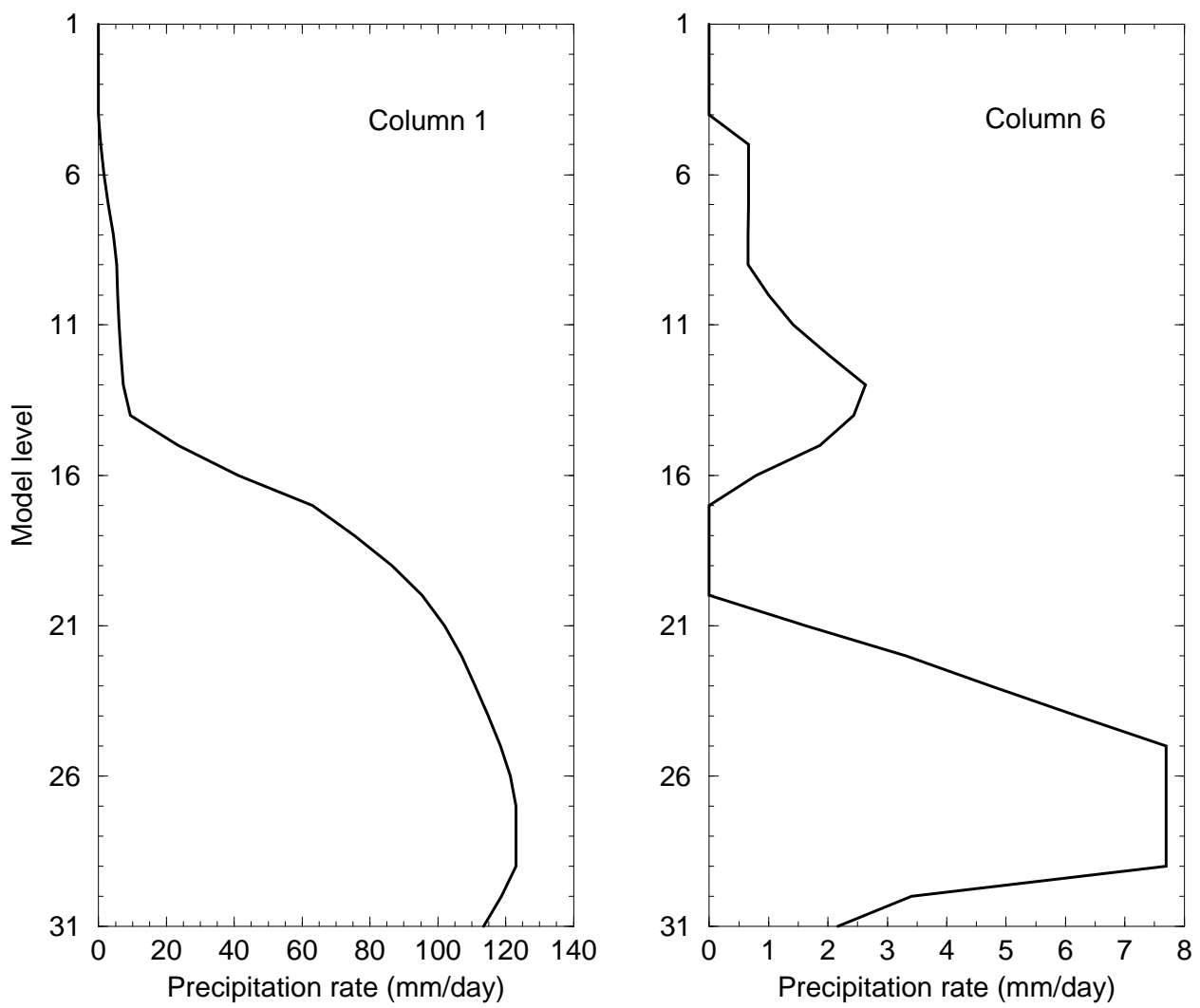

Figure 6. Vertical distribution of precipitation in sub-columns one (left panel) and six (right panel) of the sub-grid precipitation model. The values refer to precipitation leaving the base of a given model level.

which evaporation can occur as $a_{P}^{k}-a^{k}$ assuming maximum overlap between precipitation and cloud. Secondly it determines the "local" precipitation rate in the area that contains precipitation as $P^{k} / a_{P}{ }^{k}$, where $P^{k}$ is the grid mean precipitation rate. The T93 parametrization contains a description of $a_{P}{ }^{k}$ which can be expressed as

$$
a_{P}^{k}=\max \left\{a_{P}^{k-1},\left[\frac{a^{k} \Delta P+a_{P}^{k-1} P^{k-1}}{\Delta P+P^{k-1}}\right]\right\}
$$

where $a_{P}{ }^{k-1}$ is the area covered by precipitation leaving the level above and is thus the area covered by precipitation entering level $k . P^{k-1}$ is the amount of precipitation leaving level $k-1, \Delta P$ is the amount of precipitation generated in level $k$, and $a^{k}$ is the cloud fraction at level $k$. Evaporation of precipitation does not alter $a_{P}$, except when precipitation evaporates completely.

Figure 7 compares the precipitation fraction diagnosed from the sub-grid model with that given by (7). The parametrization following (7) does not correctly capture the vertical distribution of $a_{P}$. There are two major differences; in the anvil region (model level 11 to 16), the precipitation fraction is underestimated by (7) whereas below those levels it is considerably overestimated. The reasons for these differences lie in the construction of (7). In this parametrization, $a_{P}{ }^{k}$ is determined as a weighted average of the precipitation fraction of level $k-1$ and the cloud fraction in level $k$, where the weights are the precipitation flux in layer $k-1$ and the change in precipitation flux due to microphysical processes in layer $k$. This weighting was introduced to prevent levels that do not contribute to precipitation from being contributors to $a_{P}$. In the example shown, this parametrization underestimates $a_{P}$ in the anvil part since the precipitation added to the flux in that part $(\Delta P)$ is not large enough compared to the incoming flux from above $(P)$ to increase the precipitation fraction to the correct value of 1 . However, as is shown below, a more important difference is the overestimation of $a_{P}$ below level 16. This difference results from the maximum statement in (7), which prevents a reduction in $a_{P}$ unless all precipitation evaporates. This impacts the evaporation of precipitation, since the area over which precipitation is allowed to evaporate is defined as $a_{P}{ }^{k}-a^{k}$.

Figure 8 compares the grid mean evaporation rates of the sub-grid and the T93 model. The grid-mean 


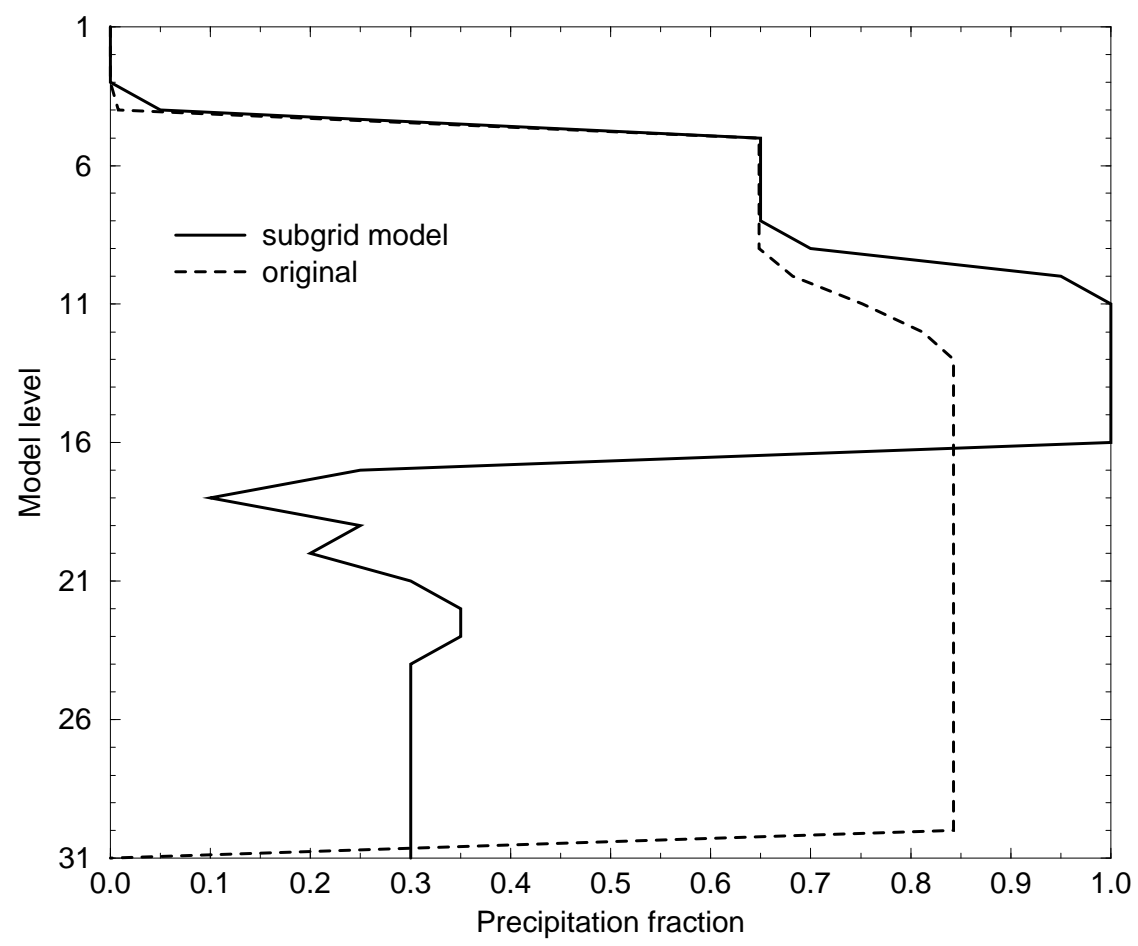

Figure 7. Precipitation fraction as simulated by the sub-grid precipitation model (solid) and the T93 scheme (dashed). The values refer to the fraction covered by precipitation at the bottom of a given model level.

values for the sub-column model are calculated by averaging over the values of the individual sub-columns. As expected, large differences exist in the middle troposphere with a strong overestimation of evaporation by the original scheme. The most likely explanation of this difference is that an overestimation of $a_{P}$ overestimates the amount of precipitation available for evaporation. To test whether this is the major source of the difference, the simulation of the original scheme is repeated with the precipitation fraction prescribed to be that diagnosed from the sub-grid precipitation model. The results are shown in Figure 8 (dot-dashed curve). At least in this case, the overestimation of $a_{P}$ causes most of the large differences in evaporation of precipitation in the middle troposphere. However, there are still major differences such as that at model level 16.

It is worthwhile investigating the cause for this difference, since model level 16 is the only level where the "true" $a_{P}$ is much larger than the cloud fraction, so that a significant area of the grid-box is subject to evaporation of precipitation. Because the formula for evaporation depends on the local precipitation rate in the volume undergoing evaporation of precipitation, an overestimation of evaporation could result from an overestimation of the precipitation rate in this volume. Figure 9 shows the distribution of the precipitation rate in model level 16 for the sub-grid model and the T93 scheme with prescribed $a_{P}$, just before the parametrization of evaporation is calculated. Since $a^{16} \approx 0.07$, only the first sub-column is assumed to be cloudy while $a_{P}{ }^{16}=1$. It is evident that for most of the sub-columns undergoing evaporation (columns 2 through 20 ) the precipitation rate before evaporation (solid curve) is lower than the mean precipitation rate given by the T93 scheme with prescribed $a_{P}$ (dashed curve). In fact, excluding the sub-column with the largest precipitation rate (column 1), which is not subject to evaporation since it is cloudy, the average precipitation rate of the sub-grid model is 5.8 $\mathrm{mm} /$ day as compared to $7.9 \mathrm{~mm} /$ day for the T93 model. This difference directly contributes to the difference in evaporation rate at level 16. This suggests a very important error source in the T93 parametrization even with a perfect simulation of $a_{P}{ }^{k}$, namely that the local precipitation rate in the region undergoing evaporation maybe significantly less than the grid-mean precipitation flux divided by the precipitation fraction. Using only a grid averaged precipitation flux implies averaging the flux inside the cloud, where it increases downwards due to conversion and accretion, with that outside the cloud which decreases downwards due to evaporation. This 


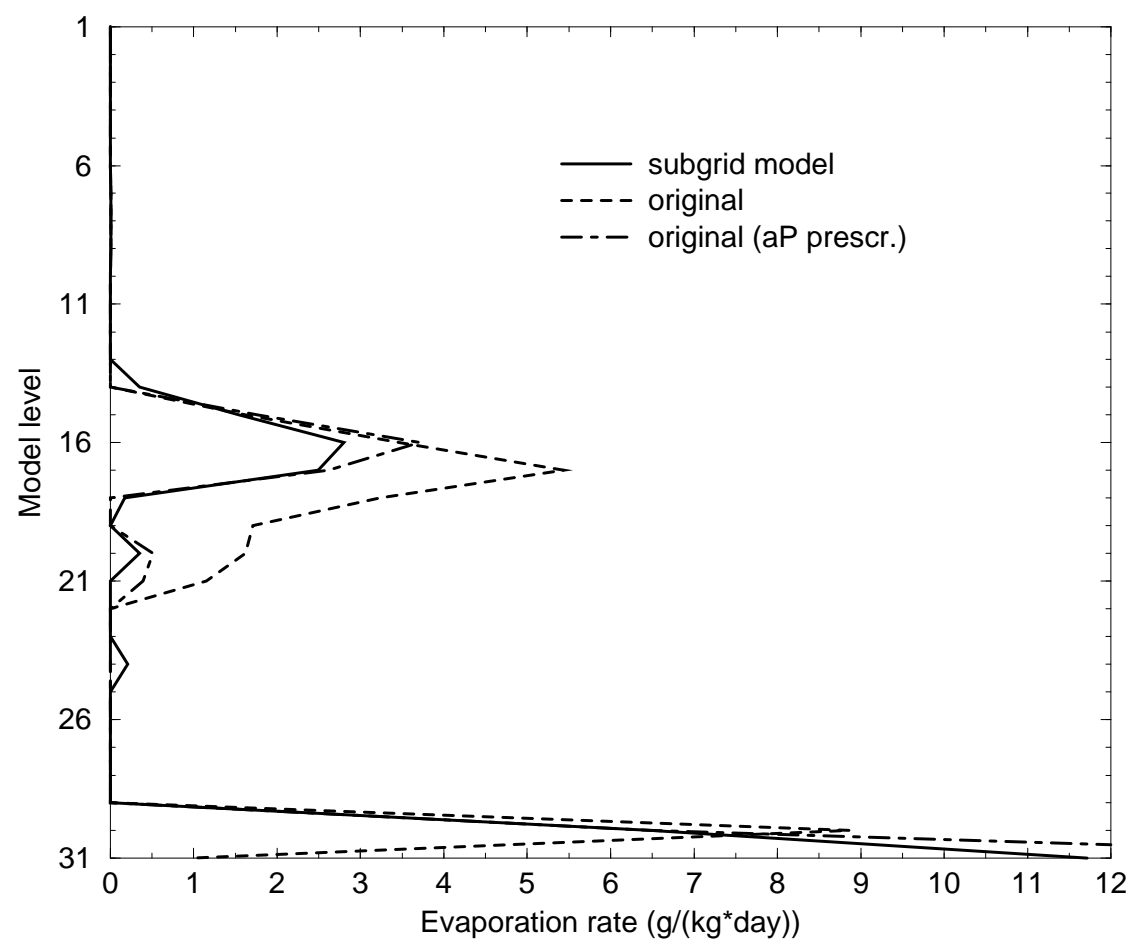

Figure 8. Evaporation rate as simulated by the sub-grid precipitation model (solid), the T93 scheme (dashed), and the T93 scheme with prescribed $a_{P}$ (dot-dashed)

averaging leads to an artificial horizontal transport of precipitation from the generally larger values inside cloud to the generally smaller values outside cloud and thereby to the observed overestimation of evaporation. This problem will tend to occur at all cloudy levels with precipitation and $a_{P}{ }^{k}>a^{k}$.

Figure 10 compares the grid-mean precipitation flux for the same simulations as in Figure 8. Large differences between the sub-grid and the T93 model begin at level 16 and increase downwards. The precipitation reaching the surface and hence the net latent heating of the column differ by more than $8 \mathrm{~mm} /$ day with no precipitation at all reaching the surface in the original scheme. Forcing the precipitation fraction to be that diagnosed from the sub-grid model alleviates most of the discrepancies except near level 16.

\section{(b) Global model}

Several errors in the parametrization of microphysical processes that result from improperly accounting for the sub-grid scale distribution of cloud and precipitation have been identified for a single case. To assess the generality of these results, the global ECMWF model is integrated for one timestep $(\Delta t=60 \mathrm{~min})$ at spectral resolution T63. The reason for using only one timestep is again to establish the direct effect of the sub-grid model on the model physics without allowing feedbacks to occur. The precipitation fraction, evaporation rate, and precipitation rate are compared using the sub-grid precipitation model and the current T93 for the initial conditions of the ECMWF operational model on 12UTC 1 July 1997. The initial distribution of cloud fraction for this time was shown in Figure 2.

Figure 11 compares the zonal mean of the precipitation fraction as analysed from the simulation with the sub-grid model to that of the T93 scheme. The differences between the two models are large with the most significant differences occurring in the Tropics. Similar to the single column case, the T93 scheme underestimates high level $a_{P}$, but largely overestimates midlevel $a_{P}$. The differences in the extratropical regions are smaller, although the sub-grid model yields a smaller $a_{P}$ in the middle troposphere. In the extratropics one could speculate that vertically varying cloud fraction plays a smaller role because cloud fraction is more uniform, e.g. in frontal cloud systems. 


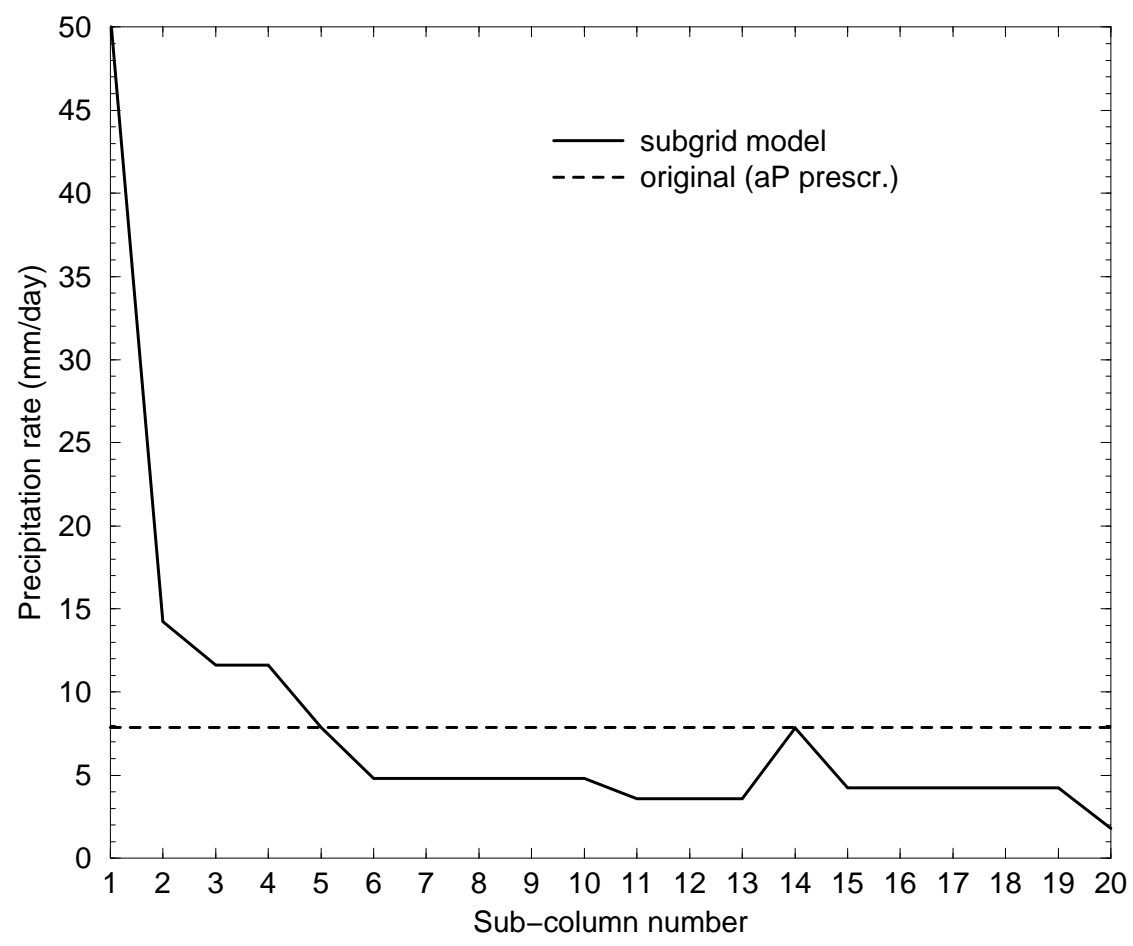

Figure 9. Precipitation rate at the top of model level 16 as simulated by the sub-grid precipitation model (solid) and the T93 scheme with prescribed $a_{P}$ (dashed)

With errors in $a_{P}$ similar to those in the single column simulation, similar errors in the grid-mean evaporation and precipitation rates can also be expected for the global model. Figure 12 shows the zonal mean of the grid mean evaporation rate for both the T93 and the sub-grid model, and Figure 13 the zonal mean surface large-scale precipitation. The strong overestimation of evaporation in the middle troposphere in the Tropics is evident and leads to a difference of up to $1.5 \mathrm{~mm} /$ day in zonal mean large-scale precipitation, equivalent to more than $40 \mathrm{~W} / \mathrm{m}^{2}$ of latent heat release.

Table 1 summarizes the global mean values for some of the components of the hydrological cycle for the first time step. Starting from the same initial liquid water/ice content $(0.126 \mathrm{~mm})$ both models generate about the same amount of precipitation $(\approx 2.65 \mathrm{~mm} /$ day $)$. However, the evaporation rate for the sub-grid model is about $20 \%$ smaller than that of the T93 model leading to about $0.2 \mathrm{~mm} /$ day more large-scale precipitation. Since the difference in the net latent heat release due to large-scale precipitation constitutes an extra forcing for the model it will certainly affect the full model simulation. As this study concentrates on the direct physical effects of vertically varying cloud fraction, a discussion of the effects on the model climate is beyond its scope and will be carried out elsewhere.

\begin{tabular}{|c||c|c|}
\hline & T93 & Sub-grid \\
\hline \hline Liquid water/ice content $(\mathrm{mm})$ & 0.126 & 0.126 \\
\hline Formation of precipitation (mm/day) & 2.67 & 2.65 \\
\hline Evaporation of precipitation (mm/day) & 0.99 & 0.78 \\
\hline Surface precipitation (mm/day) & 1.68 & 1.87 \\
\hline
\end{tabular}

TABLE 1. Global means for components of the hydrological cycle (large-scale only) for the first time step of a T63L31 integration with the sub-grid precipitation model and the T93 scheme 


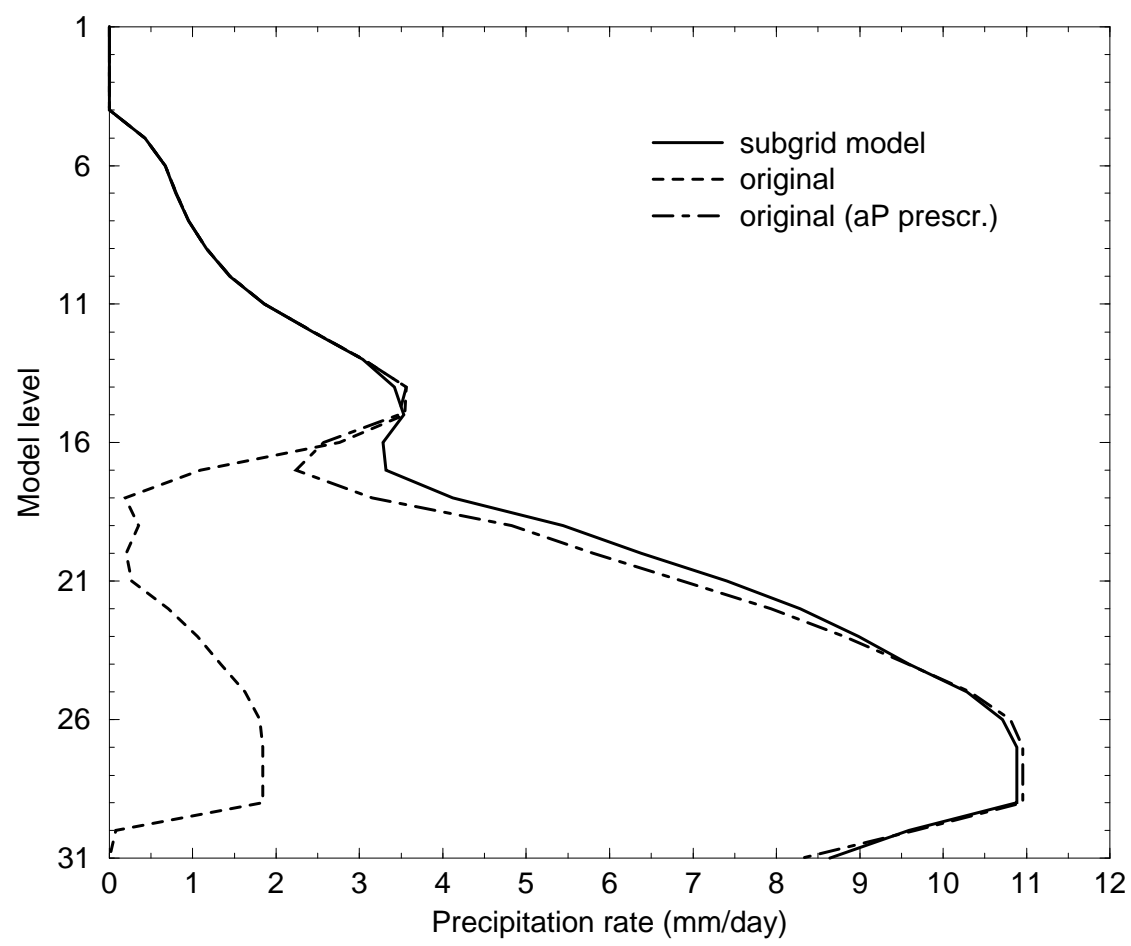

Figure 10. Precipitation rate as simulated by the sub-grid precipitation model (solid), the T93 scheme (dashed), and the T93 scheme with prescribed $a_{P}$ (dot-dashed)

Before ascribing differences between the T93 scheme and the sub-grid model to "errors" in parametrization, it is necessary to establish that the results of the sub-grid model are not sensitive to arbitrary assumptions in the construction of the model. The two main assumptions that might influence the results are the placement of cloudy boxes and the number of sub-grid columns used.

As already mentioned in section 3 the horizontal distribution of the columns in Figure 4 should not be interpreted as a spatial distribution. In fact if all the columns were redistributed randomly the result of the precipitation calculations would not change at all. It is therefore more appropriate to consider the distribution of the columns as a probability distribution rather than a spatial one. One of the constraints for the distribution is the cloud overlap assumption for the grid box. This determines how many of the cloudy sub-boxes in a model level need to be placed in columns containing clouds in at least one of the higher levels in the column, and how many in columns that contain clear sky in all higher levels of the column. However, as explained above, the exact placement within each of these two groups (referred to as "cloud under cloud" and "cloud under clear sky") is ambiguous. The distribution shown in Figure 4 and used so far in the study is built by assigning higher probabilities to certain columns to be cloudy. For instance, in the case of cloud under cloud, cloudy boxes are preferentially placed in columns that contain cloud in the layer immediately above. Furthermore for each of the two groups, the probability is artificially a function of the column index, because boxes are filled preferentially from the "left" (Figure 4). In order to study the impact of these assumptions on the grid-mean results, these preferences are removed and new clouds are placed randomly within each group. Note, however that the maximum-random cloud overlap assumption for the grid-box as a whole as expressed in (3) is retained. The precipitation rate for the one-timestep integration in the tropics differs between these two column placement options (for convenience named left and random) by about $1 \mathrm{~mm} /$ day (Figure 14). The smaller precipitation rates in the random case result from smaller accretion rates caused by the removal of the "tower" of cloud that exists in the "left" placement case in column 1 (Figure 4). The differences are however much smaller than those to the T93 parametrization (cf. Figure 10). The global model results (not shown) confirm the results of the single column simulation. 

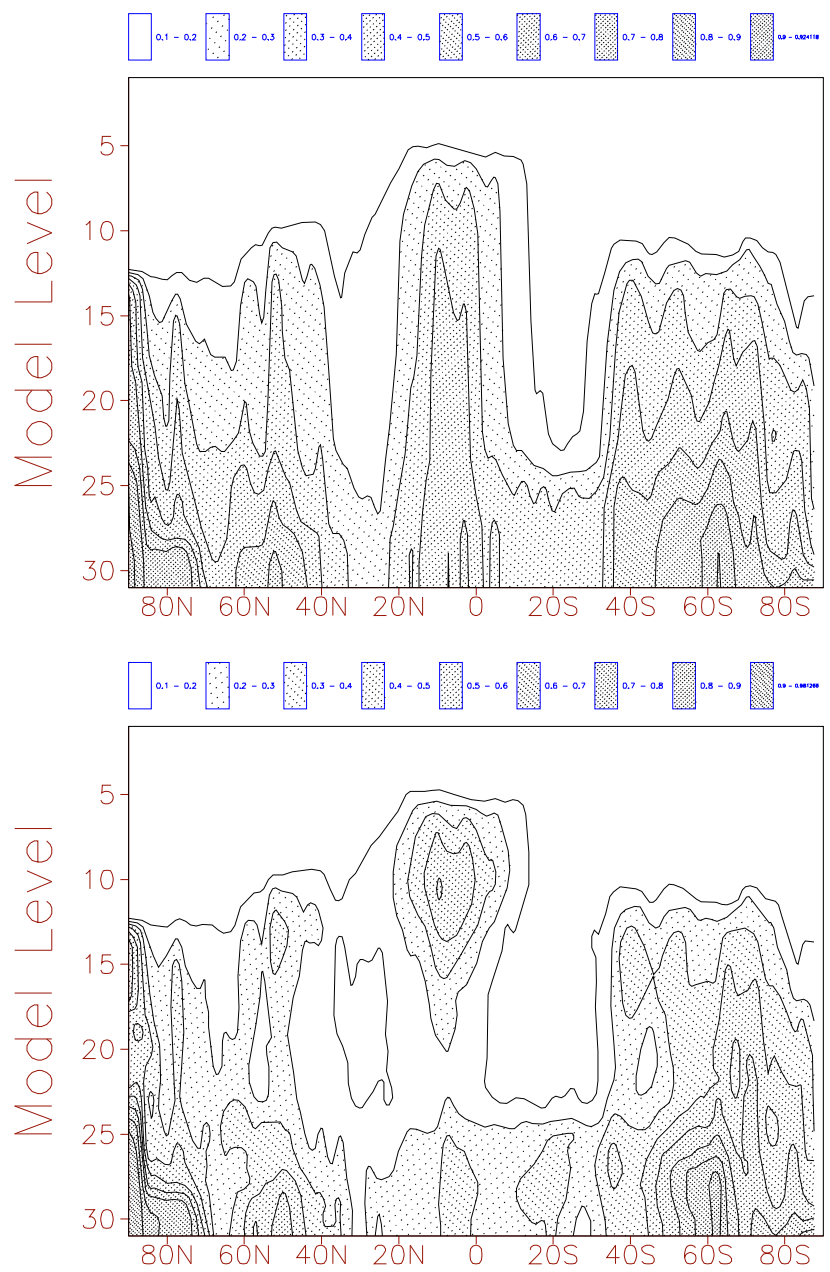

Figure 11. Zonal mean of precipitation fraction for the first time step of a T63L31 integration with the global model for 1 July 1997, 12UTC. Top: T93, bottom: Sub-grid model

Another important sensitivity test is whether the results of the sub-grid model depend on the number of sub-columns used. Since the sub-grid model rounds the actual model cloud fraction to a multiple of the sub-column size, which is obviously a function of the number of columns used, it is desirable to use as many sub-columns as possible, to minimize rounding errors. However, if one expects the model cloud fraction to be accurate to not more than 0.05 than the use of say 100 sub-columns would not be sensible. Figure 15 shows the precipitation rate for the one-timestep integration in the Tropics using 10, 20 (default) and 100 sub-columns in the sub-grid precipitation model. The differences between these simulations are smaller than the differences between simulations with different box placement (Figure 14) and certainly much smaller than the differences between the sub-grid model and the T93 scheme (Figure 10). These results are confirmed by the global model experiment (not shown).

The use of different values for specific humidity in the calculations of precipitation evaporation $\left(q_{v}\right.$ in T93 

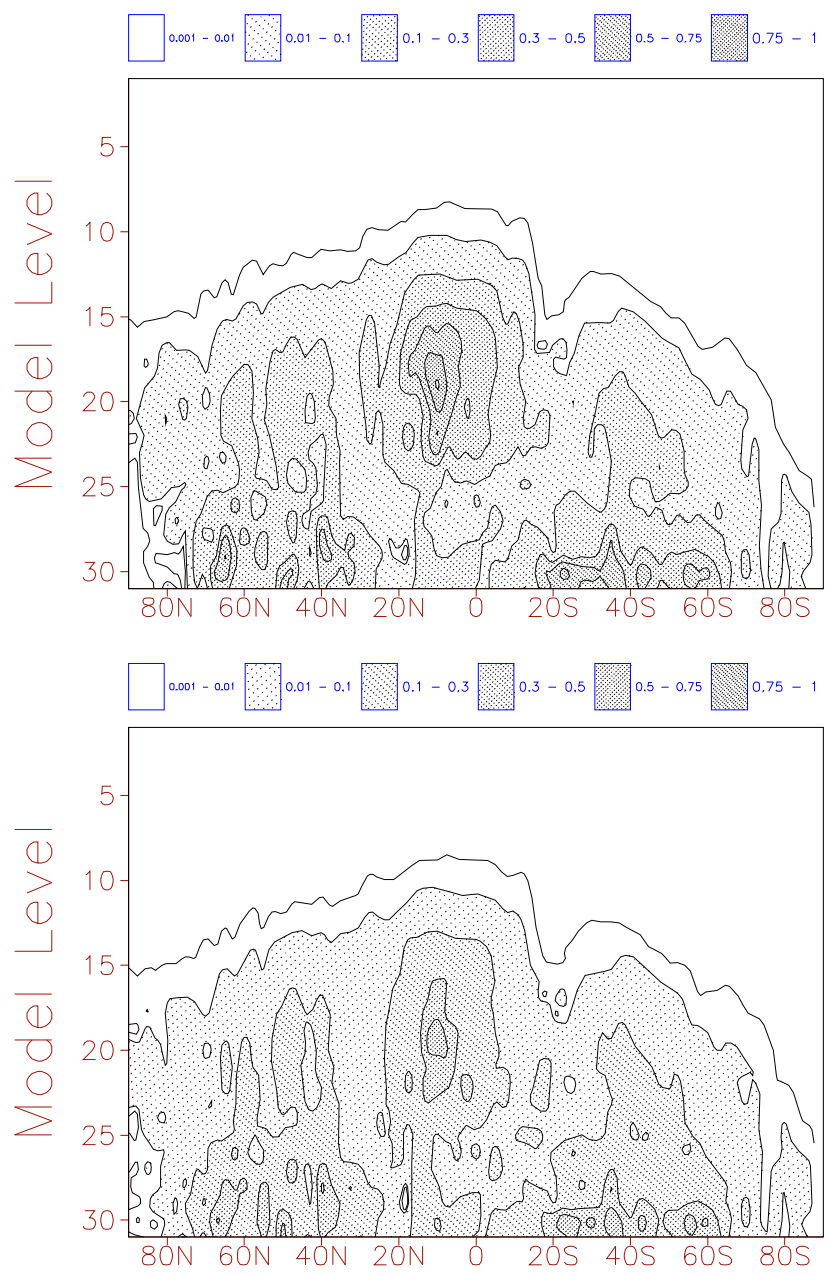

Figure 12. Zonal mean of evaporation rate in $\mathrm{g} /\left(\mathrm{kg}^{*}\right.$ day) for the first time step of a T63L31 integration with the global model for 1 July 1997, 12UTC. Top: T93, bottom: Sub-grid model

and $q_{v}{ }^{c l r}$ in the sub-grid model) might also contribute to the differences shown. Therefore the single-column calculations were repeated using $q_{v}$ in the sub-grid model and $q_{v}{ }^{c l r}$ in the T93 scheme. The results (not shown) indicate little sensitivity to the choice of specific humidity for the sub-grid model and increased evaporation rates in the T93 parametrization leading to even larger discrepancies between the two simulations. The different behaviour of the two schemes can be explained by their very different simulation of precipitation fraction. In case of the sub-grid model the precipitation fraction decreases rapidly below the anvil so that evaporation of precipitation cannot occur at all or in only few sub-columns. Hence the small sensitivity of this scheme to the choice of specific humidity. In contrast the T93 parametrization creates large areas of evaporation below the anvil because of its overestimation of precipitation fraction. Hence changing the specific humidity used in the evaporation calculations exerts a larger influence.

So far all sensitivity tests used the maximum-random overlap cloud overlap assumption of the current 


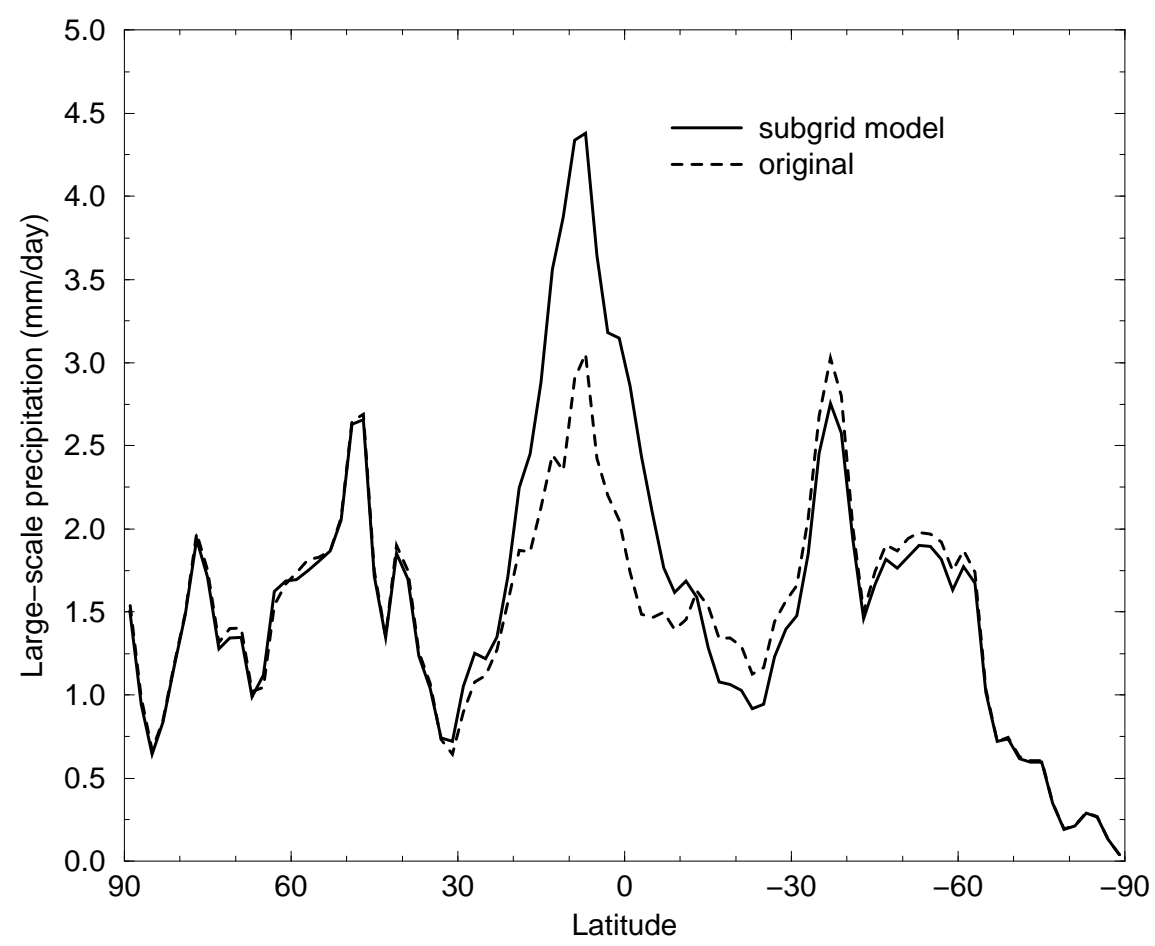

Figure 13. Zonal mean large-scale precipitation rate at the surface for the first time step of a T63L31 integration with the sub-grid precipitation model (solid) and the T93 scheme (dashed)

model. Altering this assumption will indicate what role the cloud overlap assumption will play when using a more complex treatment of vertically varying cloud fraction. It should be noted that since the T93 parametrization uses maximum-random overlap for clouds (and implicitly assumes a maximum overlap of cloud with precipitation), a comparison of the T93 scheme with the results of the sub-grid model with a different overlap assumption is not appropriate. Hence, the main purpose of this test is to establish the sensitivity of the sub-grid scheme (and hopefully parametrized cloud microphysics as well) to the cloud overlap assumption. Figure 16 shows the zonal mean precipitation of three one-timestep integrations of the global model using the sub-grid precipitation scheme with maximum-random, maximum and random overlap. All integrations use the default method ("left") to place cloudy boxes. It is evident, that a large difference exists between random overlap and the other two overlap assumptions. The reason for the large reduction in precipitation when using random overlap is the horizontal "spreading out" of the cloud which leads to higher evaporation and lower accretion rates. The implications of this result are discussed below.

\section{Summary AND Discussion}

The study uses a sub-grid precipitation model to assess the performance of the 1993 ECMWF stratiform precipitation scheme in the presence of vertically varying cloud fraction. The basic idea of the sub-grid scheme is to divide the model grid-box into several sub-columns and, after distributing the parametrized cloud such that each column is either cloudy or clear sky, to solve the microphysics part of the parametrization for each column separately. Extensive comparisons between the sub-grid model and current parametrization have been carried out in the context of one timestep experiments for both the single column model and the global model. The use of one timestep simulations has been preferred to longer model integrations in order to understand the direct implications of the different treatment of cloudiness variations in the vertical.

The comparison revealed two important shortcomings of the T93 parametrization:

- There is an erroneous simulation of precipitation fraction. 


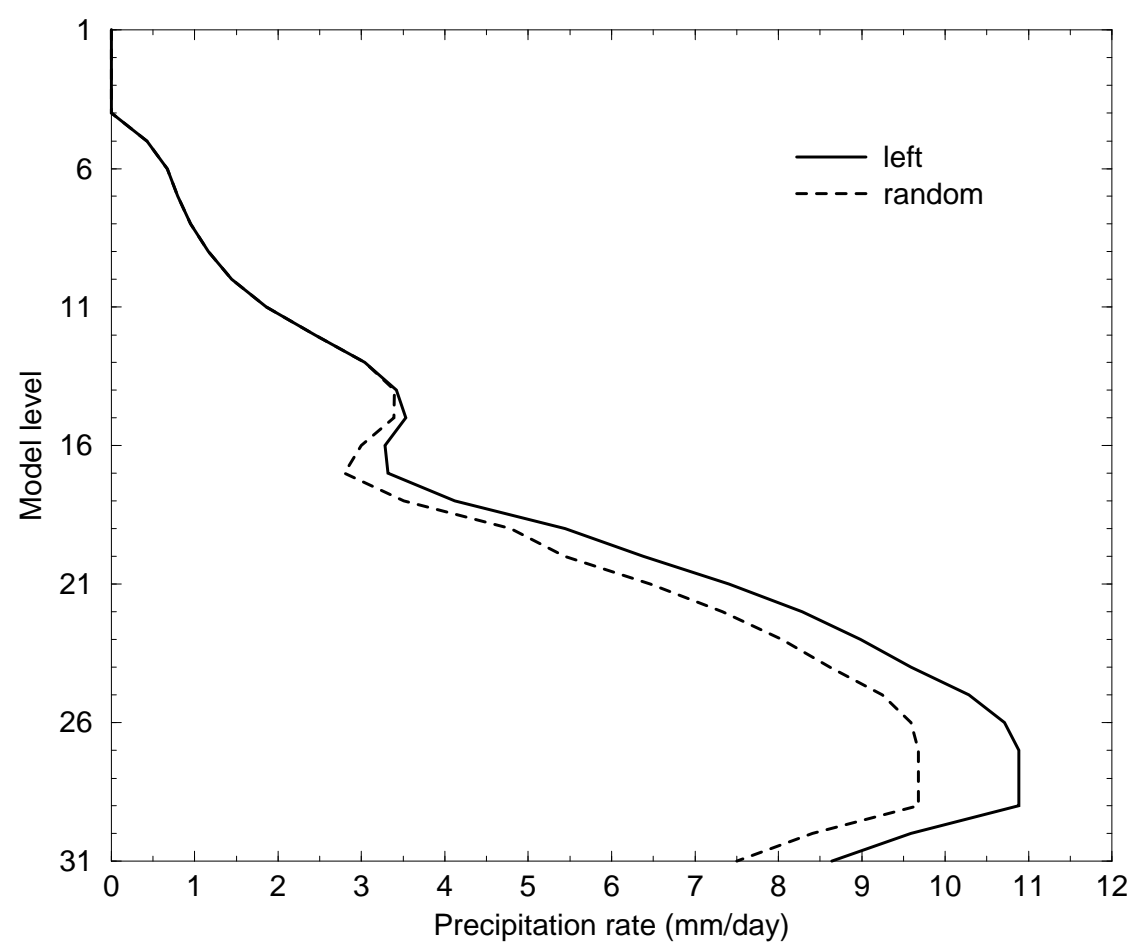

Figure 14. Precipitation rate as simulated by the sub-grid precipitation model with "left" (solid) and "random" (dashed) cloud placement. Both simulations assume the same maximum-random cloud overlap assumption.

- Large truncation errors are introduced by averaging the precipitation flux over the whole precipitation area. This average results from the assumption that the local precipitation rate equals the grid mean precipitation rate divided by the precipitation fraction.

The main effect of these errors has been found to be an overestimation of evaporation, a process whose parametrization GCMs are known to be very sensitive to. A generalization of these results to other parametrization schemes is difficult, since little is revealed in the literature about the details of the treatment of cloud and precipitation overlap when parametrizing stratiform precipitation in connection with cloud fraction. An exception is the study of Rotstayn (1997), which addresses the first item on the list above. However, to the authors knowledge, there is no parametrization that attempts to address the second item above, which can be equally important once a good parametrization of the precipitation fraction has been found.

Several sensitivity studies were carried out to estimate the influence of assumptions used in the construction of the sub-grid model. Little sensitivity has been found to the number of sub-columns used as long as the number is large enough to avoid large rounding errors. A number greater than ten appears sufficient in the ECMWF model. A slightly larger sensitivity exists to the way the sub-columns are filled with cloud once the general cloud overlap assumption has been enforced. Although larger than the sensitivity to the number of sub-columns the differences are small compared to those with the original parametrization introducing some confidence in the findings of the comparison.

A large sensitivity has however been found to the general overlap assumption. A change from maximumrandom to random overlap leads to a dramatic decrease of the stratiform precipitation rate over the whole globe. This is not surprising since the random overlap assumption maximizes the spreading out of the clouds in the grid box thereby reducing the accretion rate and increasing the evaporation rate. It is necessary to stress again that this large sensitvity does not invalidate the general results of the study, since the current parametrization uses a maximum-random overlap of clouds and therefore cannot be sensibly compared to a sub-grid scheme that uses a different cloud overlap scheme. However, the large sensitivity stresses the need for improved knowledge 


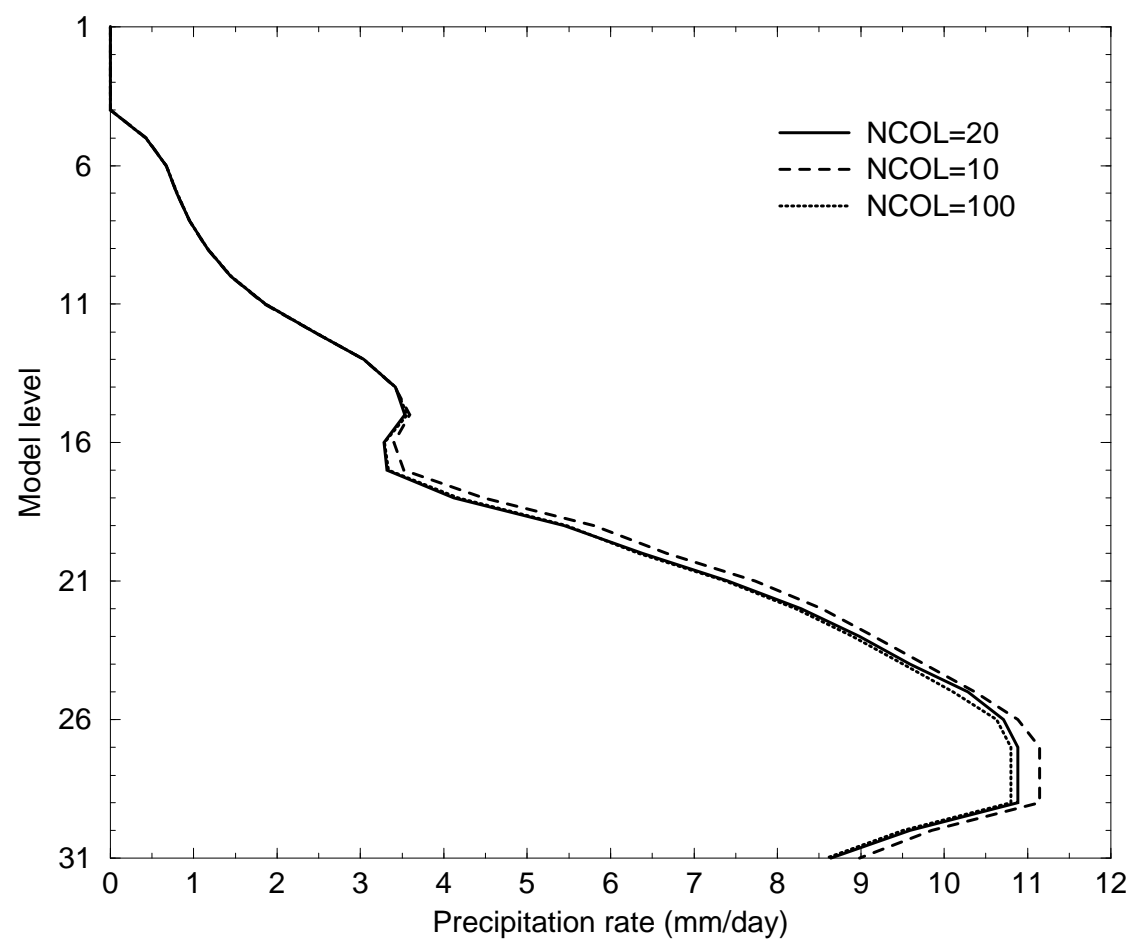

Figure 15. Precipitation rate as simulated by the sub-grid precipitation model using 20 (solid), 10 (dashed) and 100 (dotted) sub-columns

on the best way of overlapping clouds when introducing a treatment of vertically varying cloud fraction not only into the radiation but also into the microphysics parametrization. It is also noteworthy that given the relatively high vertical resolution of the ECMWF model (around $40 \mathrm{hPa}$ in the free troposphere) the random overlap assumption does not seem appropriate.

In the light of the current tendency to improve microphysical parametrizations in large-scale models by introducing more and more complex bulk microphysical schemes the results here suggest that great care has to be taken when addressing the fact that modern cloud parametrizations can produce large variations in cloud fraction in the vertical. A balance between the sophistication of the cloud microphysics and the sophistication of the cloud macrophysics (e.g. cloud and precipitation fractions and their overlaps) would seem to be prudent.

A necessary step is of course the validation of the vertical cloud variation produced by the parametrization. Little is known about the performance of cloud schemes for GCMs in that respect although a recent first study by Mace et al. (1998), which compares model clouds to radar observations at the models vertical resolution on an hourly time-scale, shows encouraging results for the ECMWF model. The study is however limited to cloud occurrence statistics at one location and in one season and therefore does not cover the variety of cloud situations encountered globally. Wang and Rossow (1995) present some general statistics on cloud layering based on radiosonde observations at 30 sites. A qualitative comparison of the ECMWF model results with their findings can be found in the Appendix. It confirms the ability of the model to produce reasonable vertical cloud structures.

Observations of precipitation fractions near the surface have recently been derived from radar measurments by Sui et al. (1997) for the TOGA/COARE area and could prove extremly useful in the validation of this crucial parameter. Further guidance is also to be expected through the intercomparisons of single-column versions of GCMs with Cloud Resolving Models (CRMs) as they are currently performed in the context of the GEWEX Cloud System Study (GCSS) or the Atmospheric Radiation Measurement (ARM) programs.

There is a variety of possible applications of the sub-grid model developed for this study. The most obvious is its direct implementation into a GCM, but this might not be practical because of the extra calculations 


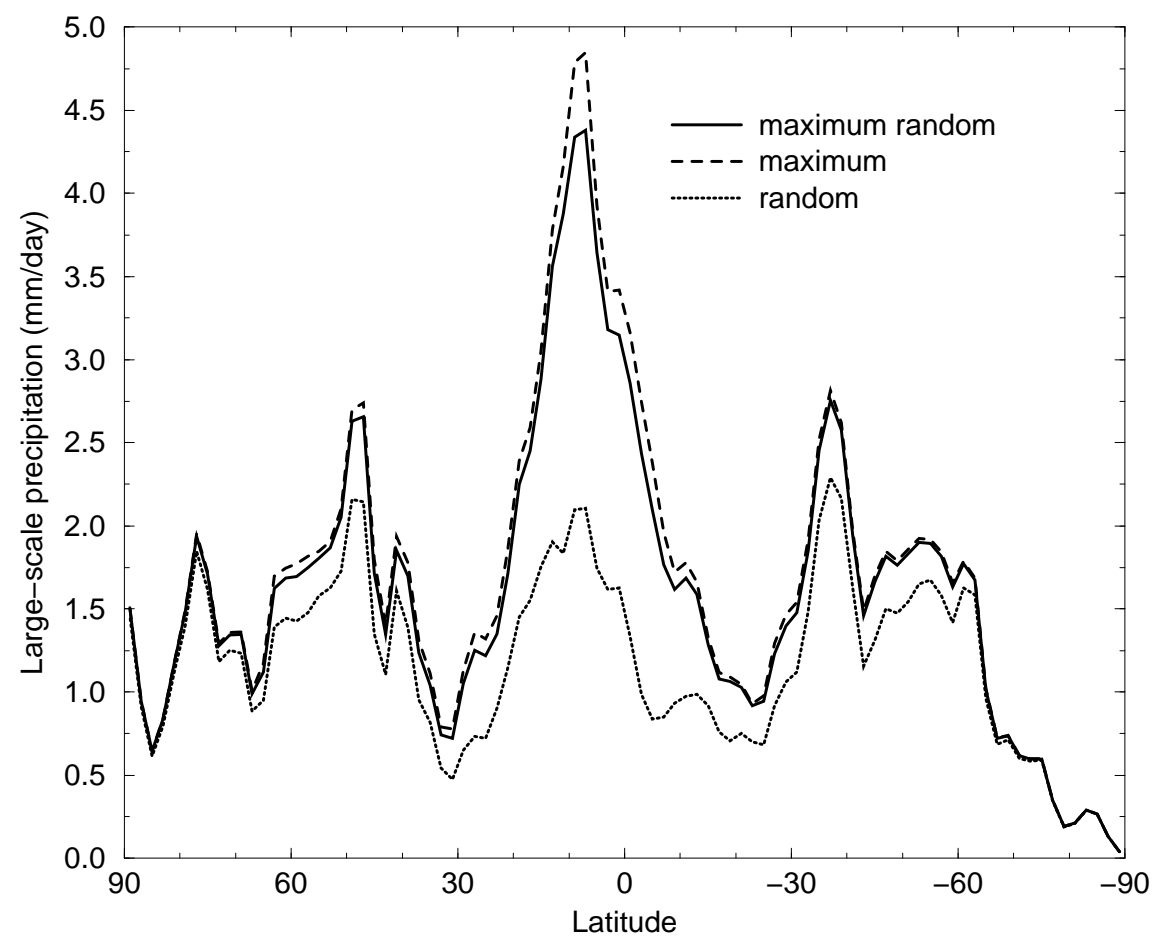

Figure 16. Zonal mean large-scale precipitation rate for the first time step of a T63L31 integration with the sub-grid precipitation model using maximum-random (solid), maximum (dashed), and random (dotted) cloud overlap

introduced by the multiple integrations of the microphysics parametrization. Adding the sub-grid model with 20 sub-columns to a T63L31 4 month integration of the ECMWF model increased the CPU time by about $10 \%$. One can speculate that this increase will be even larger when more sophisticated microphysical paramtrizations are used. Nevertheless the sub-grid model can provide guidance in the development of a parametrization of the effects discussed. The results above indicate that such a parametrization has to contain at least two components: a treatment of precipitation fraction and a treatment that avoids the averaging of the precipitation flux over the whole precipitation area, for instance by introducing separate clear-sky and cloudy precipitation fluxes.A parametrization using the results of this study has been developed at ECMWF and will be described in a future paper. This paper will also address the impact of the changes in precipitation parametrization on the model climate.

Other applications of the sub-grid model include the testing of the radiation parametrization in terms of its treatment of vertically varying cloud fraction given a fixed overlap assumption (Liang and Wang 1997). Developing suitable methods of treating cloud inhomogeneity (e.g., Tiedtke 1996) using the sub-grid model for guidance also appears as a potential application. There is no doubt that with the improvements in cloud parametrization over the last decade more thought has to and will be given to the role of variations of cloud within one grid-column and the sub-grid model presented here can be used in the development of appropriate parametrizations of their effects.

\section{ACKNOWLEDGEMENTS}

We are grateful to Drs. A. Hollingsworth, M. Miller and A. Beljaars for their comments on early versions of this manuscript. We would like to thank two anonymous referees whose reviews led to further improvement of the manuscript. 
In this appendix, the statistics of cloud vertical structure in the ECMWF model are compared to that inferred from an analysis of radiosondes presented in Wang and Rossow (1995). Histograms of the number of cloudy layers in a column, the thickness of these cloud layers, and the separation distance between cloud layers for vertical columns containing multilayered clouds are presented. To compare model data to observed it is assumed that if cloud occurs in vertically adjacent model levels then they are part of a single cloud layer. Cloud occurrence in a model level is defined as when the cloud fraction of that level is greater than a threshold value, here taken as $10^{-5}$. For example, if cloud occurs in model levels $6,7,8$, and 23, then there are 2 cloud layers with thickness 3 and 1 model levels, and a separation distance between layers of 14 model levels.

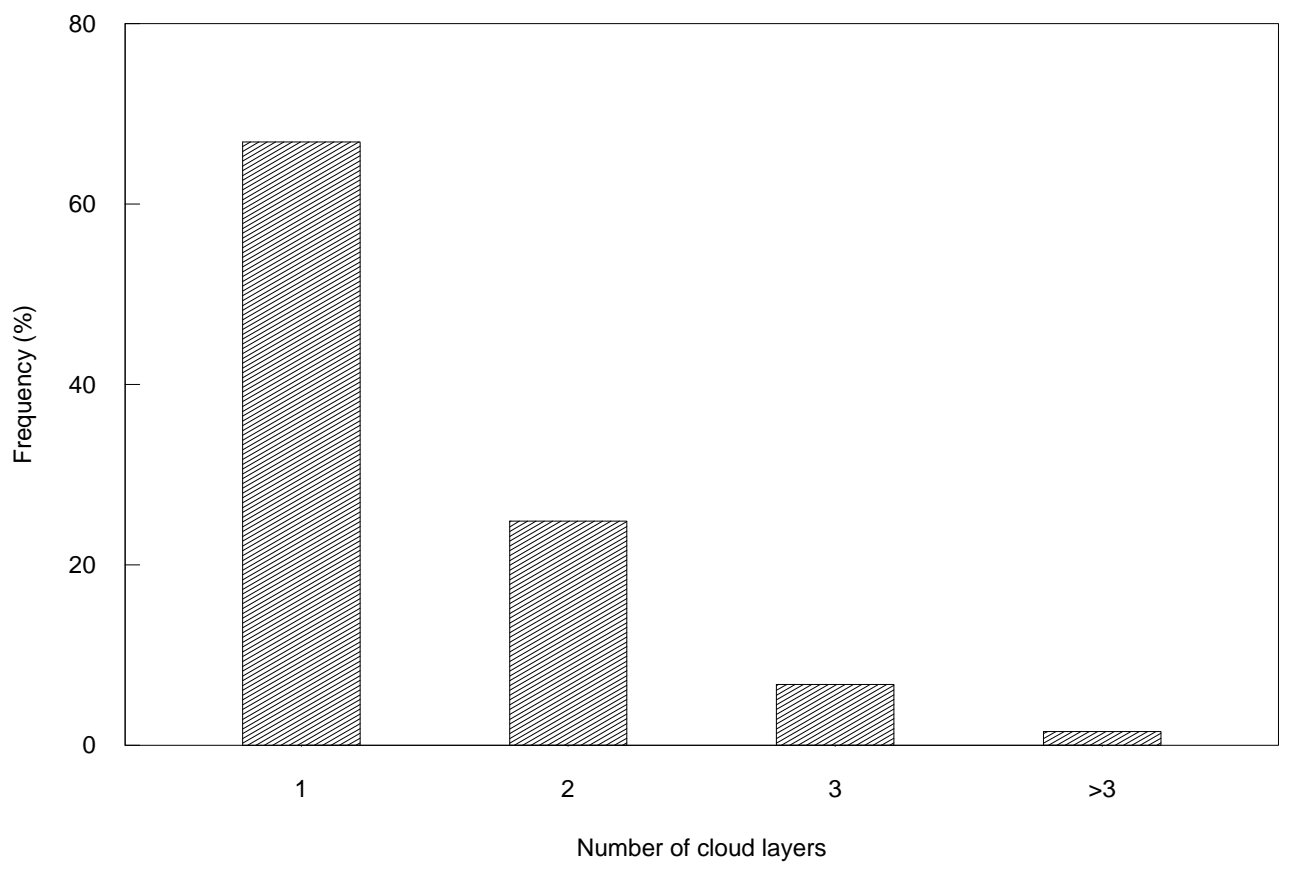

Figure A.1. Frequency distribution of the number of cloud layers in a T63L31 24h forecast

The histogram of the number of cloud layers per column (excluding columns with no clouds) is presented in Figure A.1. This histogram is taken from a 24 hour forecast of the current ECMWF model run with 31 vertical levels at T63 horizontal resolution. Approximately one-third of the cloud columns contain multi-layer clouds with the majority of multi-layered cloud columns containing two cloud layers. According to the analysis of radiosondes (Figure 18 of Wang and Rossow), in soundings with $30 \mathrm{hPa} / 50 \mathrm{hPa}$ vertical resolution multilayer cloud systems occur in 56\%/28\% respectively, of the soundings containing any cloud layers. Considering that the thickness of ECMWF model levels is about $40 \mathrm{hPa}$ in the mid-troposphere, the ECMWF model slightly underestimates the frequency of multilayer cloud systems.

Histograms of cloud thickness and separation distance are presented in Figure A.2. Qualitatively, the shape of these histograms agrees well with the observations (Figures 20 and 25, respectively, of Wang and Rossow). One important difference is that if one model level is assumed to be 500 meters thick, the histogram of cloud thickness falls off more slowly than the observations; i.e., the ECMWF model has more cloud layers of moderate to large thickness than observed. However, the thickness of cloud layers in the ECMWF model is sensitive to the threshold used to define cloud occurrence. If levels with cloud fraction less than 0.1 are excluded, the histogram of cloud thickness falls off more rapidly. The abundance of cloud fractions less than 0.1 in proximity to levels with cloud fractions greater than 0.1 has been found to be related to vertical advection of cloud fraction by the mean wind.

Statistics of cloud vertical structure resolved by the sub-grid model (i.e. Figure 4) are similar to those presented above. 


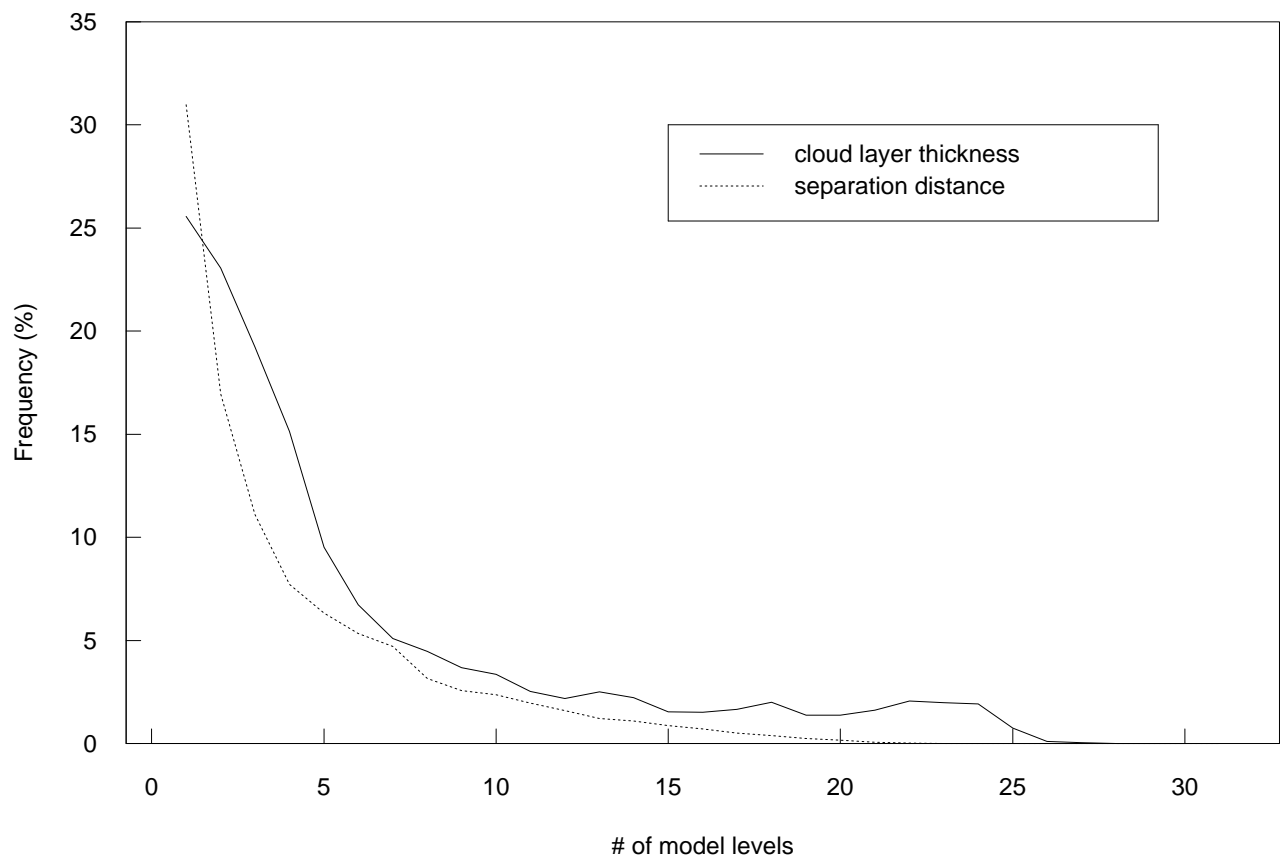

Figure A.2. Frequency distribution of the thickness and separation distance of cloud layers in a T63L31 24h forecast

\section{REFERENCES}

Baker, M. B.

Bechthold, P., Pinty, J. P. and Mascart, P.

Del Genio, A. D., Yao, M.-S., Kovari, W. and Lo, K. K.-W. Fowler, L. D., Randall, D. A. and Rutledge, S. A.

Geleyn, J.-F. and

Hollingsworth, A.

Gregory, D.

Heymsfield, A. J. and

Donner, L. J.

Houze, R. A. and Betts, A. K.

Hsie, E. Y., Farley, R. D. and Orville, H. D.

Jakob, C.

Kessler, E.

Liang, X.-Z. and Wang, W.-C.

Lin, Y.-L., Farley, R. D. and Orville, H. D.

Mace, G. G., Jakob, C. and Moran, K. P.
1997 Cloud microphysics and climate. Science, 276, 1072-1078

1993 The use of partial cloudiness in a warm-rain parameterization: A subgrid-scale precipitation scheme. Mon. Wea. Rev., 121, 3301-3311

1996 A prognostic cloud water parametrization for global climate models. J. Clim., 9, 270-304

1996 Liquid and ice cloud microphysics in the CSU general circulation model. Part I: Model description and simulated microphysical processes. J. Clim., 9, 489-529

1979 An economical analytical method for the computation of the interaction between scattering and line absorption of radiation. Beitr. Phys. Atmos., 52, 1-16

1995 A consistent treatment of evaporation of rain and snow for use in large-scale models. Mon. Wea. Rev., 123, 2716-2732

1990 A Scheme for Parameterizing Ice-Cloud Water Content in General Circulation Models. J. Atmos. Sci., 47, 1865-1877

1981 Convection in GATE. Rev. Geophys. Space Phys., 19, 541576

1980 Numerical simulation of ice phase convective cloud seeding. J. Appl. Meteor., 19, 950-977

1994 The Impact of the New Cloud Scheme on ECMWF's Integrated Forecasting System (IFS). Proceedings of ECMWF/GEWEX Workshop on Modelling, Validation and Assimilation of Clouds, ECMWF, November 1994

1969 On the distribution and continuity of water substance in atmospheric circulations. Meteor. Monogr., 10, No. 32, Amer. Met. Soc., 84 pp

1997 Cloud overlap effects on general circulation model climate simulations. J. Geophys. Res., 102, 11039-11047

1983 Bulk-parameterization of the snow field in a cloud model. J. Clim. Appl. Meteor., 22, 1065-1092

1998 Validation of hydrometeor occurrence predicted by ECMWF model using millimeter wave radar data. accepted by Geophys. Res. Letters 
Morcrette, J.-J. and

Fouquart, Y.

Rotstayn, L. D.

Rutledge, S. A. and Hobbs, P. V.

Smith, R. N. B.

Stubenrauch, C. J., Del

Genio, A. D. and Rossow, W. B.

Sui, C.-H., Lau, K.-M.,

Takayabu, Y. N. and

Short, D. A.

Sundqvist, H.

Sundqvist, $\mathrm{H}$.

Tian, L. and Curry, J. A.

Tiedtke, M.

Tiedtke, M.

Wang, J. and Rossow, W. B.

Yu, W., Doutriaux, M., Sèze, G., LeTreut, H. and M. Desbois
1986 The overlapping of cloud layers in shortwave radiation parameterizations. J. Atmos. Sci., 43, 321-328

1997 A physically based scheme for the treatment of stratiform clouds and precipitation in large-scale models. I: Description and evaluation of the microphysical processes. Quart. J. Roy. Met. Soc., 123, 1227-1282

1983 The mesoscale and microscale structure and organization of clouds and precipitation in midlatitude cyclones. VIII: A model for the "seeder-feeder" process in warm-frontal rainbands. J. Atmos. Sci., 40, 1185-1206

1990 A scheme for predicting layer clouds and their water content in a general circulation model. Quart. J. Roy. Met. Soc., 116, 435-460

1997 Implementation of subgrid cloud vertical structure inside a GCM and its effect on the radiation budget. J. Clim., 10, 273-287

1997 Diurnal variations in Tropical oceanic cumulus convection during TOGA COARE. J. Atmos. Sci., 54, 639-655

1978 A Parameterization Scheme for Non-Convective Condensation Including Prediction of Cloud Water Content, Quart. J. Roy. Met. Soc., 104, 677-690

1988 Parameterization of Condensation and Associated Clouds in Models for Weather Prediction and General Circulation Simulation, Physically-Based Modelling and Simulation of Climate and Climate Change, M.E. Schlesinger, Ed., Kluwer, 433-461

1989 Cloud overlap statistics. J. Geophys. Res., 94, 9925-9935

1993 Representation of Clouds in Large-Scale Models, Mon. Wea. Rev., 121, 3040-3061

1996 An extension of cloud-radiation parameterization in the ECMWF model: The representation of subgrid-scale variations of optical depth. Mon. Wea. Rev., 124, 745-750

1995 Determination of cloud vertical structure from upper-air observations. J. Appl. Meteor., 34, 2243-2258

1996 A methodology study of the validation of clouds in GCMs using ISCCP satellite observations. Clim. Dyn., 12, 389401 